\title{
Explicit extraction of absorption peak positions, widths and heights using higher order derivatives of total shape spectra by nonparametric processing of time signals as complex damped multi-exponentials
}

\author{
Dževad Belkić1,2 . Karen Belkić1,2,3,4
}

Received: 10 December 2017 / Accepted: 30 December 2017 / Published online: 19 January 2018 (C) The Author(s) 2018. This article is an open access publication

\begin{abstract}
Sole lineshape estimation of non-parametrically computed higher-order derivatives of spectral envelopes in different modes (complex, real, imaginary, magnitude) is investigated. The processed time signals are sums of complex attenuated exponentials (harmonics). The peak parameters of the derivative spectra are directly connected to those of the customary (non-derivative) absorption lineshapes. Crucially, this permits the reconstruction of the latter from the former parameters (the latter are sought since they are unknown, whereas the former are extractable from the derivative envelopes). The cross-checking relationships of the lineshapes for the magnitude modes with the real and imaginary parts of the complex-valued envelopes (total shape spectra) are established. The explicit procedure and the necessary analytical expressions are presented for reconstruction of the exact locations, widths and heights of all the retrieved physical resonances (spectral peaks). These facets are illuminated in the derivative fast Padé transform (dFPT) using its non-parametric version, i.e. without solving the quantification problem (no polynomial rooting, no tackling of eigen-value problems, etc.). Two kinds of illustrations for derivative spectra are reported. One deals with the general Breit-Wigner resonance formula and its first three derivatives. The other is concerned with the dFPT in clinical diagnostics of relevance to breast
\end{abstract}

$\triangle$ Dževad Belkić

Dzevad.Belkic@ki.se

1 Department of Oncology-Pathology, Karolinska Institute, Building P-9, 2nd floor, P.O. Box 260, 17176 Stockholm, Sweden

2 Medical Radiation Physics and Nuclear Medicine, Karolinska University Hospital, Stockholm, Sweden

3 School of Community and Global Health, Claremont Graduate University, Claremont, CA, USA

4 Institute for Prevention Research, Keck School of Medicine, University of Southern California, Alhambra, CA, USA 
cancer detection by magnetic resonance spectroscopy. A systematic parallel between these two examples is drawn to highlight, in a stepwise manner, the role of paramount importance played by derivative lineshapes, especially for disentangling overlapping resonances that invariably plague all quantitative analyses of spectra.

Keywords Magnetic resonance spectroscopy $\cdot$ Breast cancer diagnostics $\cdot$ Mathematical optimization - Fast Padé transform - Derivative spectra

\section{Abbreviations}

$\begin{array}{ll}\text { ADC } & \text { Analogue-to-digital converter } \\ \text { AR } & \text { Auto-regression } \\ \text { ARMA } & \text { Auto-regressive moving average } \\ \text { au } & \text { Arbitrary units } \\ \text { BW } & \text { Bandwidth } \\ \text { dFFT } & \text { Derivative fast Fourier transform } \\ \text { dFPT } & \text { Derivative fast Padé transform } \\ \text { dMRS } & \text { Derivative magnetic resonance spectroscopy } \\ \text { dMRSI } & \text { Derivative magnetic resonance spectroscopic imaging } \\ \text { FFT } & \text { Fast Fourier transform } \\ \text { FID } & \text { Free induction decay } \\ \text { FPT } & \text { Fast Padé transform } \\ \text { FWHM } & \text { Full-widths at half-maximum } \\ \text { g } & \text { Gram } \\ \text { HLSVD } & \text { Hankel-Lanczos singular value decomposition } \\ \text { Hz } & \text { Hertz } \\ \text { Lac } & \text { Lactate } \\ \text { MA } & \text { Moving average } \\ \text { MHz } & \text { Megahertz } \\ \text { MR } & \text { Magnetic resonance } \\ \mu \text { M } & \text { Micromole } \\ \text { mM } & \text { Millimole } \\ \text { MRI } & \text { Magnetic resonance imaging } \\ \text { MRS } & \text { Magnetic resonance spectroscopy } \\ \text { MRSI } & \text { Magnetic resonance spectroscopic imaging } \\ \text { NMR } & \text { Nuclear magnetic resonance } \\ \text { PA } & \text { Padé approximant } \\ \text { PC } & \text { Phosphocholine } \\ \text { PE } & \text { Phosphoethanolamine } \\ \text { ppm } & \text { Parts per million } \\ \text { s } & \text { Second } \\ \text { SNR } & \text { Signal-to-noise ratio } \\ \text { SRI } & \text { Spectral region of interest } \\ \text { SVD } & \text { Singular value decomposition } \\ \text { T } & \text { Tesla } \\ \text { ww } & \text { Wet weight } \\ & \end{array}$




\section{Introduction}

A very common stumbling block in signal processing across inter-disciplinary fields of scientific, as well as technological/industrial research and development, is the occurrence of unresolvent components in compound spectral peaks [1]. Processed time signals, irrespective of their origin, are given in their ubiquitous forms of linear combinations of exponentials. These can be either oscillatory (i.e. containing sine and cosine functions of time) or non-oscillatory (i.e. without such trigonometric functions). Both variants are encompassed in complex damped exponentials. An example of spectral crowding is the abundance of closely overlapped peaks generated from time signals encoded by conventional magnetic resonance spectroscopy (MRS) used, e.g. in medicine through cancer diagnostics [2].

A number of procedures exist in attempts to tackle this difficult problem, with varying chances of success. Primarily due to its computational efficiency, the fast Fourier transform (FFT), with the Cooley-Tukey algorithm [3] is most frequently employed to generate total shape spectra or envelopes. These, however, invariably exhibit unresolved components that the FFT cannot autonomously quantify to determine the peak positions, widths, heights and phases. To estimate the latter parameters, most techniques resort to post-processing of the envelopes from the FFT by fitting the compound peaks with several Lorentzians or Gaussians. Also, linear combinations of these two lineshapes are used in fitting as surrogates of the Voigt profile, which is a convolution via integration of the product of a Lorentzian and a Gaussian. These are all inadequate approaches. The reasons for the inadequacy of all the fitting techniques are non-uniqueness and subjectivity. Any number of components (including those of a spurious character) can be adjusted to reproduce some of the unresolved peaks. However, there is no way to separate the physical lineshapes from the non-physical component profiles that are used to merely minimize the errors of fitting by way of some, more or less, arbitrary constraints [4-6].

There are other, mathematically founded signal processors that do not rely at all upon fitting of envelopes from the FFT. Such are the methods that treat the set of time signal points as a data matrix (the Hankel matrix) to be processed by various algorithms of linear algebra. These are matrix diagonalizations, solving systems of linear equations, singular value decomposition (SVD), polynomial rooting by the equivalent Hessenberg eigen-value problem, etc. The well-known examples are, e.g. the Hankel-Lanczos SVD (HLSVD) [7] and the fast Padé transform (FPT) [1,2]. The HLSVD diagonalizes the Hankel matrix with the help of the SVD and, further, it uses the Lanczos algorithm. The HLSVD solves two systems of linear equations, one to obtain the frequencies and the other for the amplitudes. Once the latter parameters are found, the HLSVD computes the envelopes by the FFT. This is the case because the HLSVD does not have its own formula for the spectrum. The FPT first extracts the expansion coefficients of two polynomials $P_{L}$ and $Q_{K}$ using only the input time signal points by solving a single system of linear equations with an optional refinement through the SVD. This first step immediately gives the frequency-dependent spectrum as the polynomial quotient $P_{L} / Q_{K}$ which is the complex envelope in the FPT. The peak parameters are obtained subsequently by rooting the denominator polynomial $Q_{K}$. This gives the fundamental (nodal, eigen) complex frequencies. These provide 
the positions and widths of the peaks, as the real and imaginary parts of complex frequencies, respectively. Then, the Cauchy residue analytical formula of $P_{L} / Q_{K}$ comes into play, to yield the complex amplitudes. Each of these latter quantities is a pair of a magnitude and a phase, as the absolute value and argument (angle) of a complex amplitude. The ratios of the magnitude of the amplitude and the lineshape widths produce the peak heights. The resonance peak area of an absorptive Lorentzian is the halved absolute value of the amplitude of the given component of the input time signal. The concentration of a component metabolite in MRS is directly proportional to the corresponding peak area. Such a determination of the peak positions, widths, heights and phases in the FPT completes the linelist of the resonance parameters obtained by explicitly solving the quantification problem, which is equivalently called the problem of spectral analysis. The number $K$ of physical resonances, as yet another unknown quantity, is the number of stable components. Further, unlike the HLSVD, the fast Padé transform, on its own, possesses a number of different expressions for the frequency spectrum. For example, the non-parametric FPT gives the envelope as the rational function $P_{L} / Q_{K}$. On the other hand, the parametric FPT yields the same spectrum as the sum of the Heaviside partial fractions. This latter spectrum can also be written in the canonical form involving only the roots of $P_{L}$ and $Q_{K}$, as yet another Padé-based response function. The success of the parametric, quantification version of the FPT has been abundantly documented in the MRS literature both for simulated (noiseless and noisy) and encoded time signals [1,2,8-25].

Of late, the non-parametric variant of the FPT has been promoted from its customary lineshape estimation to the stage of component retrieval. To this end, two different strategies have been introduced, one qualitative and the other quantitative. The former is the partitioned FPT [26,27], whereas the latter is the derivative fast Padé transform (dFPT) [28]. The partitioned FPT, through the two separate contributions to the non-parametric envelope $P_{L} / Q_{K}$, can visualize the hidden components of compound peaks. Here, one contribution to $P_{L} / Q_{K}$ comes from its moving average (MA) part, and the other is due to the remaining auto-regressive (AR) process. The two constituent partitions, AR and MA, form the combined process known as auto-regressive moving average (ARMA). The so-called $z$-transform of the time-domain ARMA process gives the rational response function $P_{L}(z) / Q_{K}(z)$, which is the FPT [1]. The quantity $z$ is the complex frequency dependent harmonic variable. The partitioned FPT is qualitative, since it does not yield the accurate numerical values of peak parameters. Although the partitioned FPT can split apart overlapped resonances, it cannot reconstruct the exact quantitative characteristics of the visualized peaks. Nevertheless, the partitioned FPT is useful as it can give an initial hint into the substructure of compound peaks. In fact, this option is automatically built into the Padé algorithm to provide the very first output. In other words, the algorithm of the FPT runs several pathways in concert, from the partitioned spectra to quantification with the linelist of spectral parameters.

The other non-parametric processing, which can both visualize and exactly quantify all the recovered single, isolated peaks as well as the components of compound resonances, is the derivative fast Padé transform, dFPT. This variant has recently been introduced within MRS, where it was shown that high-order derivatives in the dFPT can reconstruct the exact input peak parameters, despite using only estimation of lineshapes of spectral envelopes [28]. No solution of the quantification problem, and 
no envelope fitting either, is involved in the dFPT. All that is needed is to first nonparametrically compute the Padé-Green function $G(v) \equiv P_{L}(v) / Q_{K}(v)$, where $v$ is the linear real-valued sweep frequency. This is followed by the application of the differential transform $(\mathrm{d} / \mathrm{d} \nu)^{m}(m=1,2,3, \ldots)$ to $G(v)$ yielding the $m$ th derivative spectrum $G^{(m)}(v)=(\mathrm{d} / \mathrm{d} v)^{m} P_{L}(v) / Q_{K}(v)$.

In the present work, the dFPT is further explored. The goal is to provide the complete procedure with the explicit theoretical expressions that enable the attainment of the exact peak parameters (position, width, height) from the reconstructed envelope lineshapes in the dFPT, especially with high derivative order $m$. The significance of this goal would be best appreciated if the unknown non-derivative $(m=0)$ absorption peak parameters could be deduced from the corresponding extracted derivative $(m \geq 1)$ peak parameters of real, imaginary and magnitude lineshapes. This is a key to signal processing since the absorptive, symmetric, bell-shaped Lorentzian peak is the most straightforward for analysis and interpretation. The practical usefulness of this complete procedure in the dFPT is illuminated for early tumor diagnostics by single-voxel derivative magnetic resonance spectroscopy (dMRS). Also highlighted are the advantages offered by the dFPT in processing time signals for volumetric coverage of the examined tissue by multi-voxel magnetic resonance spectroscopic imaging (MRSI). When equipped by production of derivative spectra, this modality becomes the derivative magnetic resonance spectroscopic imaging (dMRSI). The new nomenclature dMRS and dMRSI were first coined in Ref. [28].

\section{Theory}

\subsection{Use of external perturbations of generic systems to elicit their internal structure}

The analog (continuous) time signal $c(t)$ and the corresponding frequency spectrum $G(v)$ are two dual, equivalent representations of the same information. In MRS, and indeed throughout nuclear magnetic resonance (NMR) spectroscopy, the time signals are the input data from either encoding (measurements) or computing (theory). Both the time, $t$, and the linear frequency, $v$, are continuous independent variables of functions $c(t)$ and $G(v)$, respectively. These independent variables are expressed in units of seconds (s) and hertz (Hz), respectively. The time signal $c(t)$ is equivalently called the free induction decay (FID) response. The clause "response" transcends the physical meaning of $c(t)$. This is reflected already in the understanding that a response is ordinarily made when a question is asked. A question comes as a kind of "perturbation" which triggers an answer. Such an intuitively self-evident notion, taken for granted in everyday life, is also ingrained in the scientific quest concerning the structure of matter. Matter, in its equilibrium with the environment, would not spontaneously reveal its structure. Only when externally perturbed would the matter respond. It is through a response to an external perturbation that the matter would uncover some (or all) of its internal structure. Thus, generally, one needs to perturb a system to tease out its composition or structure. How much of the matter's structure could be deciphered depends on the number of degrees of freedom (or kinds of motions) affected by the 
external perturbation. The outside agents with their energies imparted onto the system set its constituents in motion through various types of "oscillations" (rotations, vibrations, ...). Since all matter is built from molecules, atoms and their components (electrons, nuclei, elementary particles,...), the response of the perturbed system crucially depends on the perturbing energy whose extent determines which distances can be probed. The larger the imparted energy, the deeper the penetrated distance. When the studied matter is human tissue, the external perturbations can be ionizing $(\mathrm{X}$-rays,...) or non-ionizing (electromagnetic fields, ultrasound,...). Interactions of sufficiently intense ionizing radiation with biological systems can cause severe damage. Individual ionizing events can involve enough energy absorbed by the cell to disrupt chemical bonds within the constituent molecules (called metabolites if the biological system is tissue). This is the reason why human exposure to ionizing radiation should be minimized. By contrast, repeated exposures of humans to scanning is permitted by non-invasive MRS, MRSI and anatomical magnetic resonance imaging (MRI) in the clinical setting with magnetic field strength $B_{0}=1.5$ or 3 tesla (T) or even stronger. The reason is that in these clinical scanners, the triple magnetic fields (static, gradient and radiofrequency pulses) are of a weak intensity, which is below the excitation thresholds of the tissue's molecules. This is why the enumerated magnetic resonance (MR) diagnostic modalities can be used for frequent monitoring of patients, initial diagnostics, post-therapeutic follow-up as well as for screening, e.g. for early tumor detection.

In MRS, a single voxel of the scanned tissue is excited by the mentioned external fields. The tissue's reaction to these external perturbations induces a current in the coil surrounding the part of the examined human body. Such a current is mapped to the analogue time signal $c(t)$. The signal $c(t)$ is subsequently switched by the analogueto-digital convertor (ADC) to its discretized counterpart $c_{n}$. In this way, the continuous variable $t$ becomes discretized via $t=n \tau(0 \leq n \leq N-1)$, where $\tau$ is the sampling rate (dwell time). The total duration $T$ of the time signal (or the total acquisition time) and the total signal length $N$ are related by $T=N \tau$. The scanned tissue slice is excited over a fixed bandwidth (BW), which is selected according to the desired part of the spectrum $G(v)$ under study. In MRS, encoded FIDs are complex-valued numbers, in which case $\tau$ and BW are inversely proportional, with unity as the proportionality constant, i.e. $\tau=1 / \mathrm{BW}$. The $\mathrm{BW}$ in $\mathrm{Hz}$ gives $\tau$ directly in $\mathrm{s}$.

\subsection{From time signals to frequency spectra and vice versa}

The duality of $c(t)$ and $G(v)$ involves the two conjugate variables, $t$ and $v$. The dual time-frequency representation implies that $G(v)$ and $c(t)$ are unambiguously obtainable from each other. Such representations with continuous $t$ and $v$ are given by the integral transforms of the type:

$$
G(v)=\int_{0}^{\infty} \mathrm{d} t \mathrm{e}^{-2 \pi i v t} c(t) .
$$


Here, the total duration $T$ of $c(t)$ is equal to infinity, $T=\infty$. In MRS, as stated earlier, the time signal $c(t)$ is acquired (encoded), whereas the frequency spectrum $G(v)$ can be in principle computed from (1). In practice, however, the digital set $\left\{c_{n}\right\}$ is finite $(N<\infty)$, and the integral in (1) is truncated at $t=T$. Irrespective of whether $t \in[0, \infty]$ or $t \in[0, T](T<\infty)$, the time signal $c(t)$ can be unequivocally reconstructed from $G(v)$ by the inverse integral transformation:

$$
c(t)=\int_{0}^{\infty} \mathrm{d} v \mathrm{e}^{2 \pi i v t} G(v) .
$$

Such integrals, either the direct version (1), or its inverse (2), are known as the Fourier integrals or the continuous Fourier transforms. The term "transform" is very important, since it is used here with the understanding that $c(t)$ and $G(v)$ are exactly extractable from each other, without any concern about the level of noise encoded in $c(t)$ alongside the physical part of the time signal. This can be viewed as being favorable and unfavorable. It is favorable from the standpoint that switching from one to another dual representation leads to no loss of information. On the other hand, it is unfavorable because the price for this latter feature is high, as the entire noise from $c(t)$ is brought as intact to the spectrum $G(v)$ due to the linearity of the transforms (1) and (2). A practical goal, however would be to strike a delicate balance between these two "extremes", within an alternative frequency representation. First, such an alternative representation should reduce noise from $c(t)$ by passing to $G(v)$. Second, $G(v)$ should inherit the entire physical information from $c(t)$. This implies that the true, physical part of the entire content (signal + noise) from the encoded $c(t)$ should be retrievable by inverting the computed $G(v)$. As shown in Ref. [1], and more specifically in Ref. [2], regarding recovery of $c(t)$ from $G(v)$, the fast Padé transform, FPT, fully accomplishes the stated task imposed upon this more powerful alternative to the Fourier representations.

There is yet another Fourier feature which can also be considered as being favorable and unfavorable. Favorable is the Fourier universality by which general time signals can be processed. Unfavorable is the same Fourier generality, as it yields low resolution, possesses no noise suppression power and, most critically, has no autonomous quantification capabilities. These latter, well-documented drawbacks are indeed caused by the Fourier generality, mainly due to ignorance of the structure of the time signal $c(t)$. Further limitations of the Fourier analysis are restriction to periodic time signals and production of merely line or stick spectra (no intrinsic interpolation), due to the pre-assigned grid frequencies unrelated to the nature of $c(t)$. The same Fourier grid $\left(v_{k}^{F}=k \Delta v_{F} \equiv k / T, 0 \leq k \leq N-1\right)$ applies to all signals of the same duration $T$. As soon as the Fourier analysis is used for $G(v)$, the input time signal is, by definition, treated as if it were a periodic function of time, i.e. $c(t+T)=c(t)$, for $t>T$. This provides no extrapolation and, thus, no prediction of the non-encoded $c(t)$ beyond $T$. In general, practice abundantly confirms that time signals $c(t)$ experimentally measured in inter-disciplinary fields are most often not periodic. Fourier spectra exist only on superficial Fourier grid frequencies that have nothing in common with the fundamental frequencies from which $c(t)$ is built. Generally speaking, line 
spectra have vertical bars (sticks) of zero widths. In the FFT spectra, the sticks are not seen as a sequence of vertical bars stacked next to each other at the fixed discretized frequencies $v_{k}^{F}(k=0,1, \ldots, N-1)$. Rather, smooth curves are drawn to connect the tips of the sticks, while plotting the given Fourier spectrum. By contrast, the spectrum $P_{L}(v) / Q_{K}(v)$ is a function of the continuous variable $v$. Even when $v$ is necessarily discretized for plotting $P_{L}(v) / Q_{K}(v)$ versus $v$, the Padé grid frequencies can be arbitrarily dense, with the sampling rate $\Delta v$ which does not need to be equal to $\Delta v_{F}=1 / T$.

Considered strictly, a general line spectrum is associated only with for purely stationary, stable bound states (e.g. ground states of atoms/molecules) of practically infinite lifetimes. On the other hand, peaks in spectra are resonances that represent non-stationary, transient states of molecules. Such metastable states last for a given lifetime, after which they decay. This is reflected in distributions of frequencies in the spectral lineshapes, instead of the stick spectra. These distributions are mainly the Lorentzian functions that are in mathematics known as the Cauchy distributions. In physics, they are also called Breit-Wigner lineshape profiles from the theory of resonant scattering of particles and photons on general targets. The Cauchy, Lorentz and Breit-Wigner distributions coincide with one of the simplest forms of the Padé approximant (PA), symbolized as [0/2], and given by:

$$
Y_{\mathrm{A}}(v)=\frac{d_{0}}{2 \pi} \frac{\Gamma_{0}}{\left(v-v_{0}\right)^{2}+\left(\Gamma_{0} / 2\right)^{2}} \quad \text { (Absorptive Lorentzian). }
$$

It is seen that the Padé spectrum $Y_{\mathrm{A}}(v)$ depends on $v$ in a continuous way. If $Y_{\mathrm{A}}(v)$ is written in the general form $P_{L}(\nu) / Q_{K}(v)$, it would follow that $P_{0}(v)=d_{0} \Gamma_{0} / /(2 \pi)$ and $Q_{K}(v)=Q_{2}(v)=\left(v-v_{0}\right)^{2}+\left(\Gamma_{0} / 2\right)^{2}$. Here, $d_{0}$ is the amplitude, $v_{0}$ and $\Gamma_{0}$ are the resonant frequency and the full width at half-maximum (FWHM), respectively. All three parameters $v_{0}, \Gamma_{0}$ and $d_{0}$ are real-valued quantities, with the understanding that the phase of the generally complex amplitude is equal to zero. The notation $[L / K]$ is the usual symbol for the general PA as a ratio of two polynomials $P_{L}$ and $Q_{K}$ of degrees $L$ and $K$, respectively. The physical meaning of a stick or bar spectrum is that it consists of lines associated with mono-energetic (mono-chromatic) absorption or emission of radiations. These latter processes result from transitions between discrete rotational, vibrational or rovibrational molecular levels for the ground state as well as for excited states. As noted, such line spectra belong to pure bound, stable states of molecules. Metastable states are prone to decay and their spectra are not lines or sticks. Rather, due to the underlying dissipative dynamics (there is a loss of energy), the absorptive radiations cannot be mono-energetic. This implies that the resulting spectra do not have sharp lines, in lieu of which a distribution of energies (or frequencies) appears. The most typical example of such frequency distributions is the absorptive Lorentzian function (3). In other words, the Lorentzian lineshape centered at $v_{0}$ is a broadened line of breadth or full width $\Gamma_{0}$. These notions are also familiar by reference to a vibrating dipole which radiates energy according to classical physics. As a result, the vibrational amplitudes decrease. In other words, the dipole vibrations are attenuated, and the lineshape of the emitted radiation energy or frequency is the same Lorentzian distribution (3) instead of a simple mono-chromatic phenomenon. 


\subsection{Structure of time signals}

Damped oscillations are also present in the time domain when considering the FID, $c(t)$, in (2). Therein, as stated, a general time signal is a linear combination of complex attenuated exponentials that, hence, must be associated with the Lorentzian (3) from the frequency domain. To elicit this connection, we first write what has just been said regarding the universal form of $c(t)$ :

$$
\begin{aligned}
c(t) & =\sum_{k=1}^{K} d_{k} \mathrm{e}^{i \omega_{k} t}, \quad \operatorname{Im}\left(\omega_{k}\right)>0, \\
d_{k} & =\left|d_{k}\right| \mathrm{e}^{i \varphi_{k}},
\end{aligned}
$$

where $\omega_{k}$ and $d_{k}$ are, respectively, the characteristic or fundamental complex angular frequencies $\left(\omega_{k}=2 \pi v_{k}\right)$ and complex amplitudes, whose phases are denoted by $\varphi_{k}$. The total number $K$ of the fundamental harmonics (harmonic oscillations) should correspond to the total number of Lorentzians in the frequency domain.

\subsection{Complex, absorption and dispersion components and envelope spectra}

To proceed alongside the stated anticipation, the insertion of (4) into (1) gives the complex spectrum of the type:

$$
\begin{aligned}
& G(v)=\frac{1}{2 \pi i} \sum_{k=1}^{K} \frac{d_{k}}{v-\zeta_{k}-i \lambda_{k}} \quad \text { (Envelope: Heaviside partial fractions), } \\
& v_{k}=\zeta_{k}+i \lambda_{k} ; \zeta_{k}=\operatorname{Re}\left(v_{k}\right), \quad \lambda_{k}=\operatorname{Im}\left(v_{k}\right), \\
& \{\mathrm{FWHM}\}_{k} \equiv \xi_{k}=2 \lambda_{k} \quad(\text { Full width at half maximum }) .
\end{aligned}
$$

This indeed shows that the envelope $G(v)$ is the sum of $K$ complex components $G_{k}(v)$ :

$$
\begin{aligned}
G(v) & =\sum_{k=1}^{K} G_{k}(v), \\
G_{k}(v) & =\frac{1}{2 \pi i} \frac{d_{k}}{v-\zeta_{k}-i \lambda_{k}}=[0 / 1] \quad \text { (Component). }
\end{aligned}
$$

The sum in (9) over the $K$ components $G_{k}(v)$ can be carried out and the result is the para-diagonal Padé approximant symbolized as $[(K-1) / K]$ :

$$
G(v)=\frac{P_{K-1}(v)}{Q_{K}(v)}=[(K-1) / K] \quad(\text { Envelope: the Padé approximant }),
$$

where $P_{K-1}(v)$ and $Q_{K}(v)$ are polynomials in variable $v$ of degrees $K-1$ and $K$, respectively. The expansion coefficients of these polynomials are uniquely identified 
from (6) and are given in terms of the fundamental frequencies $\left\{v_{k}\right\}$ and amplitudes $\left\{d_{k}\right\}$. Note that the diagonal PA is $P_{K}(v) / Q_{K}(v)$ as written by $[K / K]$. The paradiagonal and diagonal PAs are particular cases of the PA of order or rank $\{L, K\}$ as given by $[L / K]=P_{L}(v) / Q_{K}(v)$, which forms the so-called Padé table when the polynomial degrees $L$ and $K$ are varied. With the Padé nomenclature, the component $G_{k}(v)$ is also a special case of $[L / K]$ with $L=0$ and $K=1$, i.e. $G_{k}(v)=[0 / 1]$, as stated in (10).

Inserting (5) into (10), the $k$ th component can be expressed in terms of its absorption $A_{k}(v)$ and dispersion $D_{k}(v)$ :

$$
\begin{aligned}
G_{k}(v) & =\operatorname{Re}\left(G_{k}(v)\right)+i \cdot \operatorname{Im}\left(G_{k}(v)\right), \\
\operatorname{Re}\left(G_{k}(v)\right) & =A_{k}(v) \cos \varphi_{k}+D_{k}(v) \sin \varphi_{k}, \\
\operatorname{Im}\left(G_{k}(v)\right) & =A_{k}(v) \sin \varphi_{k}-D_{k}(v) \cos \varphi_{k}, \\
A_{k}(v) & =\frac{\left|d_{k}\right|}{2 \pi} \frac{\lambda_{k}}{\left(v-\zeta_{k}\right)^{2}+\lambda_{k}^{2}} \quad \text { (Pure absorption), } \\
D_{k}(v) & =\frac{\left|d_{k}\right|}{2 \pi} \frac{v-\zeta_{k}}{\left(v-\zeta_{k}\right)^{2}+\lambda_{k}^{2}} \quad \text { (Pure dispersion). }
\end{aligned}
$$

The quantities in (15) and (16) are the pure absorption $A_{k}(v)$ and dispersion $D_{k}(v)$, respectively, since the three invoked parameters $\left\{\zeta_{k}, \lambda_{k},\left|d_{k}\right|\right\}$ are all real. Further, it is seen that for $\varphi_{k} \neq 0$, both the real $\operatorname{Re}\left(G_{k}(v)\right)$ and imaginary $\operatorname{Im}\left(G_{k}(v)\right)$ parts of $G_{k}(v)$ mix the absorption $A_{k}(v)$ and dispersion $D_{k}(v)$. However, it is possible to combine $\operatorname{Re}\left(G_{k}(v)\right)$ and $\operatorname{Im}\left(G_{k}(v)\right)$ to uniquely extract $A_{k}(v)$ and $D_{k}(v)$. This is done through multiplication of (13) and (14) by $\cos \varphi_{k}$ and $\sin \varphi_{k}$, respectively. Addition of the two resulting equations yields the absorption $A_{k}(v)$ :

$$
A_{k}(\nu)=\left(\cos \varphi_{k}\right) \operatorname{Re}\left(G_{k}(\nu)\right)+\left(\sin \varphi_{k}\right) \operatorname{Im}\left(G_{k}(\nu)\right) .
$$

Similarly, multiplication of (13) and (14) by $\sin \varphi_{k}$ and $\cos \varphi_{k}$, respectively, followed by subtraction of the first from the second ensuing equation, gives the dispersion $D_{k}(v)$ :

$$
D_{k}(\nu)=\left(\sin \varphi_{k}\right) \operatorname{Re}\left(G_{k}(\nu)\right)-\left(\cos \varphi_{k}\right) \operatorname{Im}\left(G_{k}(\nu)\right) .
$$

Likewise, inserting (12)-(14) into (9), the complex envelope $G(v)$ becomes:

$$
\begin{aligned}
G(v) & =\operatorname{Re}(G(v))+i \cdot \operatorname{Im}(G(v)), \\
\operatorname{Re}(G(v)) & =\sum_{k=1}^{K}\left[A_{k}(v) \cos \varphi_{k}+D_{k}(v) \sin \varphi_{k}\right], \\
\operatorname{Im}(G(v)) & =\sum_{k=1}^{K}\left[A_{k}(v) \sin \varphi_{k}-D_{k}(v) \cos \varphi_{k}\right] .
\end{aligned}
$$

This shows that the real $\operatorname{Re}(G(v))$ and imaginary $\operatorname{Im}(G(v))$ parts of the complex $G(v)$ are not the sum of the pure $K$ absorptions $A_{k}(v)$ and dispersions $D_{k}(v)$. Rather, 
the two differently combined mixtures of $A_{k}(v)$ and $D_{k}(v)$ appear in $\operatorname{Re}(G(v))$ and $\operatorname{Im}(G(v))$. Thus, for $\varphi_{k} \neq 0$, the lineshapes of neither the envelopes $\operatorname{Re}(G(v))$ and $\operatorname{Im}(G(v))$ nor their components $\operatorname{Re}\left(G_{k}(v)\right)$ and $\operatorname{Im}\left(G_{k}(v)\right)$ can be thought of as being purely absorptive and dispersive Lorentzians, respectively. This will not be the case either when all the $K$ phases $\varphi_{k}(1 \leq k \leq K)$ are set to a constant angle, say $\varphi$. In this special circumstance, the $k$-independent phase $\varphi$ still mixes $A_{k}(\nu)$ and $D_{k}(v)$ through the $\cos \varphi$ and $\sin \varphi$ terms in (20) and (21).

In fitting techniques from MRS, the real part of the given complex total shape spectrum in the FFT is used in trying to crudely reconstruct the fundamental parameters $\left\{v_{k}, d_{k}\right\}$. However, since for complex amplitudes $d_{k}$, the phases $\varphi_{k}$ are non-zero, the real part of the FFT spectrum is not absorptive. In an attempt to obtain an approximately positive-definite envelopes, the complex spectrum is modified (prior to taking its real part) through multiplications by the so-called zeroth- and/or first-order phase corrections $\left(\mathrm{e}^{i \phi_{0}}, \mathrm{e}^{i \phi_{1}}\right)$, respectively. For example, using the zeroth-order phase correction, (19)-(21) become:

$$
\begin{aligned}
\mathrm{e}^{i \phi_{0}} G(v) & =\operatorname{Re}\left(\mathrm{e}^{i \phi_{0}} G(v)\right)+i \cdot \operatorname{Im}\left(\mathrm{e}^{i \phi_{0}} G(v)\right), \\
\operatorname{Re}\left(\mathrm{e}^{i \phi_{0}} G(v)\right) & =\sum_{k=1}^{K}\left[A_{k}(v) \cos \left(\varphi_{k}+\phi_{0}\right)+D_{k}(v) \sin \left(\varphi_{k}+\phi_{0}\right)\right], \\
\operatorname{Im}\left(\mathrm{e}^{i \phi_{0}} G(v)\right) & =\sum_{k=1}^{K}\left[A_{k}(v) \sin \left(\varphi_{k}+\phi_{0}\right)-D_{k}(v) \cos \left(\varphi_{k}+\phi_{0}\right)\right] .
\end{aligned}
$$

Thus, in general, just like the unmodified (20) and (21), the zeroth-order phase correction $\mathrm{e}^{i \phi_{0}}$ of the complex spectrum $G(v)$ cannot give $\operatorname{Re}\left(\mathrm{e}^{i \phi_{0}} G(v)\right)$ and $\operatorname{Im}\left(\mathrm{e}^{i \phi_{0}} G(v)\right)$ as purely absorptive and dispersive envelopes, respectively. This is possible only in a special, idealized case when all the fundamental phases $\varphi_{k}$ are made equal to the same constant angle $\varphi$ equal to $-\phi_{0}$. Namely, for $\varphi_{k} \equiv \varphi=-\phi_{0}(1 \leq k \leq K)$, it follows from (23) and (24) that $\operatorname{Re}\left(\mathrm{e}^{i \phi_{0}} G(v)\right)$ and $\operatorname{Im}\left(\mathrm{e}^{i \phi_{0}} G(v)\right)$ are absorptive (positive-definite) and dispersive envelopes, respectively:

$$
\begin{aligned}
& \operatorname{Re}\left(\mathrm{e}^{i \phi_{0}} G(v)\right)=\sum_{k=1}^{K} A_{k}(v), \quad \varphi_{k}=-\phi_{0} \quad(1 \leq k \leq K), \\
& \operatorname{Im}\left(\mathrm{e}^{i \phi_{0}} G(v)\right)=-\sum_{k=1}^{K} D_{k}(v), \quad \varphi_{k}=-\phi_{0} \quad(1 \leq k \leq K) .
\end{aligned}
$$

Of course, in reality, the angle correction $-\phi_{0}$ cannot ever simultaneously match all the phases $\varphi_{k}(1 \leq k \leq K)$, since generally $\varphi_{k^{\prime}} \neq \varphi_{k}\left(k^{\prime} \neq k\right)$. It is for this reason that $\phi_{0}$ (with or without $\phi_{1}$ ) would never be able to yield $\operatorname{Re}\left(\mathrm{e}^{i \phi_{0}} G(v)\right)$ as a positive-definite lineshape throughout the spectral range of interest (SRI).

When the linelist of two complex parameters $\left\{v_{k}, d_{k}\right\}$ for the $k$ th resonance, or equivalently, of four real parameters $\left\{\zeta_{k}, \lambda_{k} ;\left|d_{k}\right|, \varphi_{k}\right\}$ has been reconstructed, one can choose any desired spectral mode for presentation. To this end, positive-definite spectra 
are generally more amenable to visualization, interpretation and further analysis. Since such spectra are not the real parts of complex envelopes for $\varphi_{k} \neq 0$, one is directed towards some judicious combinations of the components $\operatorname{Re}(G(v))$ and $\operatorname{Im}(G(v))$. Thus, for $\varphi_{k} \neq 0$, one can consider such combinations from, e.g. (17) and (18) to define an alternative envelope, say $\tilde{G}(v)$ as:

$$
\begin{aligned}
\tilde{G}(v) & =\operatorname{Re}(\tilde{G}(v))+i \cdot \operatorname{Im}(\tilde{G}(v)) \\
\operatorname{Re}(\tilde{G}(v)) & =\sum_{k=1}^{K}\left[\left(\cos \varphi_{k}\right) \operatorname{Re}\left(G_{k}(v)\right)+\left(\sin \varphi_{k}\right) \operatorname{Im}\left(G_{k}(v)\right)\right] \\
\operatorname{Im}(\tilde{G}(v)) & =\sum_{k=1}^{K}\left[\left(\sin \varphi_{k}\right) \operatorname{Re}\left(G_{k}(\nu)\right)+\left(\cos \varphi_{k}\right) \operatorname{Im}\left(G_{k}(v)\right)\right]
\end{aligned}
$$

The square brackets in (28) and (29) are equal to the pure absorption $A_{k}(v)$ and dispersion $D_{k}(v)$ from (15) and (16), respectively:

$$
\begin{aligned}
& \operatorname{Re}(\tilde{G}(v))=\sum_{k=1}^{K} A_{k}(v)=\frac{1}{2 \pi} \sum_{k=1}^{K} \frac{\left|d_{k}\right| \lambda_{k}}{\left(v-\zeta_{k}\right)^{2}+\lambda_{k}^{2}}, \\
& \operatorname{Im}(\tilde{G}(v))=\sum_{k=1}^{K} D_{k}(v)=\frac{1}{2 \pi} \sum_{k=1}^{K} \frac{\left|d_{k}\right|\left(v-\zeta_{k}\right)}{\left(v-\zeta_{k}\right)^{2}+\lambda_{k}^{2}} .
\end{aligned}
$$

The same results (30) and (31) are obtained by replacing $d_{k}$ with $\left|d_{k}\right|$, i.e. taking $d_{k}$ as real by setting $\varphi_{k}=0$ in (5) and (6) from the onset. Thus, the identical pure absorption total shape spectral envelopes can be constructed in three different and equivalent ways:

- $\varphi_{k} \neq 0: \operatorname{Re}(\tilde{G}(\nu)) \&$ Eqs. (27), (28),

- $\varphi_{k}=\varphi$ (constant), $1 \leq k \leq K$ :

$\operatorname{Re}\left(\mathrm{e}^{i \phi_{0}} G(v)\right), \phi_{0}=-\varphi \&$ Eqs. (23), (25),

- $\varphi_{k}=0,1 \leq k \leq K: \operatorname{Re}(G(v)) \&$ Eq. (25).

The same applies to the pure dispersion spectra for which (29) for $\varphi_{k} \neq 0$ and (31) for $\varphi_{k}=0$ give the identical results. Here, for the third possibility with the constant $\varphi_{k}(1 \leq k \leq K)$ equal to the negative zeroth-order phase $\left(-\phi_{0}\right)$, again the pure dispersion is obtained, but with changed polarity (multiplied by -1 ), as per (24) and (26). An analogous reasoning with the three possibilities also applies to the component shape spectra.

Absorptive (15) and dispersive (16) component lineshapes contain the same information. This means that the fundamental parameters $\left\{\zeta_{k}, \lambda_{k},\left|d_{k}\right|\right\}$ can be reconstructed from either absorption (15) or dispersion (16), as will be illustrated in the Results section. In particular, the linebreadth $\{\mathrm{FWHM}\}_{k}$ of the absorption $A_{k}(v)$ is already defined in (8) as twice the imaginary fundamental frequency $\lambda_{k}$, i.e. $\{\mathrm{FWHM}\}_{k}=2 \lambda_{k}$. The 
peak height $h_{k}$ of $A_{k}(v)$ follows from (15) at $v=\zeta_{k}$ via:

$$
\left.h_{k}=\frac{1}{2 \pi} \frac{\left|d_{k}\right|}{\lambda_{k}} \quad \text { (Peak height of } A_{k}(v)\right) .
$$

The peak area $a_{k}$ under the absorptive Lorentzian profile $A_{k}(v)$ from (15) is:

$$
a_{k}=\frac{\left|d_{k}\right|}{2} .
$$

In MRS, components are assigned to metabolites. The concentration $C_{k}$ of the $k$ th metabolite is proportional to the peak area $a_{k}$, i.e. $C_{k} \sim a_{k}$. Here, the proportionality constant is the number $n_{k}$ of the resonating MR sensitive nuclei in the excited slice of the tissue. The resulting product $n_{k} a_{k}$ needs to be calibrated to have the proper units:

$$
C_{k}=n_{k} a_{k} C_{\text {ref }}
$$

where $C_{\text {ref }}$ is the concentration of the reference molecule (which is a matter of choice, e.g. water or any other substance not necessarily present in the tissue). Metabolite concentration $C_{k}$ from (37) is expressed in the same units as $C_{\text {ref }}$, which is usually given in millimole $(\mathrm{mM})$ or micromole $(\mu \mathrm{M})$ per gram $(\mathrm{g})$ of wet weight $(\mathrm{ww})$ of the scanned tissue. The running $(v)$ and the fundamental $\left(v_{k}\right)$ frequencies are counted from the resonant frequency (taken as zero) of the reference molecule.

\subsection{Magnitude mode for component and envelope spectra}

The magnitude mode for the component and total shape spectra are the absolute values $\left|G_{k}(\nu)\right|$ and $|G(v)|$ of their complex counterparts $G_{k}(\nu)$ and $G(v)$, respectively:

$$
\begin{aligned}
\left|G_{k}(v)\right| & =\frac{1}{2 \pi} \frac{\left|d_{k}\right|}{\left[\left(v-\zeta_{k}\right)^{2}+\lambda_{k}^{2}\right]^{1 / 2}}, \\
|G(v)| & =\frac{1}{2 \pi}\left|\sum_{k=1}^{K} \frac{d_{k}}{v-\zeta_{k}-i \lambda_{k}}\right| .
\end{aligned}
$$

The absolute value $\left|d_{k}\right|$ of $d_{k}$ and the peak position $\zeta_{k}$ are the same in the magnitude $\left|G_{k}(v)\right|$ and absorption $A_{k}(v)$ modes. However, the peak breadth is broadened by a factor of $\sqrt{3}$ in the magnitude mode ("Magn") relative to its absorptive ("Absorp") counterpart:

$$
\{\mathrm{FWHM}\}_{k}^{\mathrm{Magn}}=\sqrt{3}\{\mathrm{FWHM}\}_{k}^{\text {Absorp }} .
$$

\subsection{The mathematical reason for overlapping resonances}

To re-emphasize, as soon as a total shape spectrum is mentioned in MRS and beyond, the problem of utmost importance arises, and that is the abundance of resonances 
that overlap with each other. The critical aspect of this obstacle occurs when the peak-to-peak distance (chemical shift-wise in MRS) is shorter than the individual linebreadths (FWHM-wise) of the adjacent resonances. The mathematical reason for the emergence of overlapped peaks in envelopes with e.g. Lorentzian lineshapes $G(v)$ is rooted in the asymptotic behavior of the corresponding components $G_{k}(v)$ for those sweep frequencies $v$ that are far away from the nodal chemical shifts $\zeta_{k}$. This is seen by extracting the leading term from the binomial series for the reciprocal of the frequency denominator in (10):

$$
\begin{aligned}
G_{k}(v) & =\frac{d_{k}}{2 \pi i} \frac{1}{v-\zeta_{k}}\left(1-\frac{i \lambda_{k}}{v-\zeta_{k}}\right)^{-1} \\
& =\frac{d_{k}}{2 \pi i}\left[\frac{1}{v-\zeta_{k}}+\frac{i \lambda_{k}}{\left(v-\zeta_{k}\right)^{2}}+\frac{\left(i \lambda_{k}\right)^{2}}{\left(v-\zeta_{k}\right)^{3}}+\ldots\right]
\end{aligned}
$$

In this development, when the running frequency $v$ increases relative to the fixed $\zeta_{k}$ and with $\lambda_{k} \lesssim \zeta_{k}$, the higher-order terms $\left(i \lambda_{k}\right)^{l} /\left(\nu-\zeta_{k}\right)^{l+1}$ for $l \geq 1$ are negligible compared to $1 /\left(v-\zeta_{k}\right)$. In such a case, the entire content from the square brackets in (41) is dominated by the leading term $1 /\left(v-\zeta_{k}\right)$. This gives the sought asymptote of $G_{k}(v)$ as:

$$
G_{k}(v) \sim \frac{d_{k}}{2 \pi i} \frac{1}{v-\zeta_{k}} \quad\left(v \text { distant from } \zeta_{k} \text { and } \zeta_{k} \lesssim \lambda_{k}\right)
$$

However, the function $1 /\left(v-\zeta_{k}\right)$ itself decreases very slowly with the augmented $v$. The asymptote $1 /\left(v-\zeta_{k}\right)$ generates long, symmetric tails on both sides of $\zeta_{k}$ and, consequently, leads to a broad base, i.e. a wide lower part of the lineshape or the peak profile. Thus, it is the asymptotic behavior (42) which, in an envelope $G(v)$ from (9) with $K$ components $G_{k}(v)$, represents the mathematical reason for the existence of overlapped resonances. With this explanation, it is clear that especially intense components $G_{k}$ may have more extended wings that, in turn, can substantially alter (through constructive and/or destructive interference effects) even some distant weaker resonances, let alone the neighboring smaller peaks. This becomes very problematic for understanding spectral envelopes and for identifying the physical components, without which no meaningful assignment can be made with the known metabolites in MRS.

\subsection{Derivative spectra}

\subsubsection{Complex mode of derivative spectra}

The abundance of overlapped resonances in spectral envelopes impacts directly and adversely on resolution. Thus, resolution enhancement (as the capacity to separate overlapped peaks) rests upon the possibility of lineshape narrowing. The analysis from subsection 2.6 shows that the asymptotic behavior (42) generates broader peak baselines that, in turn, lead to overlapped resonances. Therefore, one of the possibilities 
for reducing such spectral crowding is to diminish the elevation of the tails from the background on both sides of resonating frequency $\zeta_{k}$. The merit of the asymptote (42) is in pointing towards a way to achieve this goal consisting of finding a lineshape which, in its lowest order, falls off as $\sim 1 /\left(v-\zeta_{k}\right)^{2}$, or faster as $\sim 1 /\left(v-\zeta_{k}\right)^{m}$ with $m>2$, at frequencies $v$ that are distant from $\zeta_{k}$. With a sufficiently high $m$, the leading asymptotic term $\sim 1 /\left(v-\zeta_{k}\right)^{m}$ could localize any original Lorentzian to a wave-packet type lineshape narrowed to the immediate neighborhood of the resonant position $\zeta_{k}$. This would amount to a powerful separation of the adjacent components that were masked in the seed envelope (6) in any clustered peak with slow decaying component asymptote $\sim 1 /\left(v-\zeta_{k}\right)$ of $G_{k}(v)$ from (10).

As just rationalized, one way to diminish the tails/wings of the given Lorentzian lineshape is secured by raising the power of the denominator in (10) for each component $G_{k}(v)$. This is precisely what the $m$ th derivative $(\mathrm{d} / \mathrm{d} v)^{m} G_{k}(v)$ offers by its mathematical form:

$$
\begin{aligned}
G_{k}^{(m)}(v) & \equiv \frac{\mathrm{d}^{m}}{\mathrm{~d} v^{m}} G_{k}^{(m)}(v) \quad(\text { The } m \text { th derivative component } k), \\
G_{k}^{(m)}(v) & =\frac{d_{k}}{2 \pi i} \frac{(-1)^{m} m !}{\left(v-\zeta_{k}-i \lambda_{k}\right)^{m+1}} \quad(m=1,2, \ldots) .
\end{aligned}
$$

The binomial expansion of the $m$ th derivative Padé spectrum $G_{k}^{(m)}(v)$ for $v$ departing from $\zeta_{k}$ and with $\lambda_{k} \lesssim \zeta_{k}$ gives the series:

$$
\begin{aligned}
G_{k}^{(m)}(v) & =\frac{d_{k}}{2 \pi i} \frac{(-1)^{m} m !}{\left(v-\zeta_{k}\right)^{m+1}}\left(1-\frac{i \lambda_{k}}{v-\zeta_{k}}\right)^{-m-1} \\
& =\frac{d_{k}}{2 \pi i}(-1)^{m} m !\left[\frac{1}{\left(v-\zeta_{k}\right)^{m+1}}+(m+1) \frac{i \lambda_{k}}{\left(v-\zeta_{k}\right)^{m+2}}+\ldots\right]
\end{aligned}
$$

The leading term in the square bracket of (45) is $1 /\left(v-\zeta_{k}\right)^{m+1}$ and, thus, for $v$ distant from $\zeta_{k}$, it follows:

$$
G_{k}^{(m)}(v) \sim \frac{d_{k}}{2 \pi i} \frac{(-1)^{m} m !}{\left(v-\zeta_{k}\right)^{m+1}} \quad(m=1,2, \ldots) .
$$

Here, even the first derivative $(m=1)$ via $G_{k}^{(1)}(\nu)$ decreases faster as $\sim\left[i d_{k} /(2 \pi)\right] /$ $\left(\nu-\zeta_{k}\right)^{2}$ than its non-derivative (or zeroth-order, $\left.m=0\right)$ counterpart $\sim\left[d_{k} /(2 \pi i)\right] /$ $\left(v-\zeta_{k}\right)$ in $G_{k}^{(0)}(v) \equiv G_{k}(v)$ from (42). Moreover, the implication from the Lorentzian derivative asymptote (46) is: the higher the derivative order $m$, the stronger lowering of the lineshape tails. Note that even though we explicitly write $m>0$ in (44) and (45), the non-derivative case with $m=0$ is also included therein.

The maximum of the $m$ th order derivative complex component spectrum $G_{k}^{(m)}(v)$ is attained for $v=\zeta_{k}$ in (44): 


$$
\begin{aligned}
H_{k, m} & \equiv \max \left\{G_{k}^{(m)}(\nu)\right\}=G_{k}^{(m)}\left(\zeta_{k}\right), \\
H_{k, m} & =\frac{m !}{2 \pi i^{m}} \frac{d_{k}}{\lambda_{k}^{m+1}} .
\end{aligned}
$$

\subsubsection{Real and imaginary modes of derivative spectra}

Alternatively, the derivative spectrum $G_{k}^{(m)}(v)$ from sub-section 2.7.1 can be generated by applying the operator $(\mathrm{d} / \mathrm{d} \nu)^{m}$ not directly to the complex profile $G_{k}(\nu)$ from (10), as we have done in (44), but to its equivalent combination of absorption $A_{k}(v)$ and dispersion $D_{k}(v)$ from (15) and (16) that are the PAs of the rank [0/2] and [1/2], respectively. In other words, the degree of the numerator polynomial of $D_{k}$ is larger than that of $A_{k}$ by one unit. Thus, the numerators of the $m$ th derivatives $A_{k}^{(m)}(v)=$ $(\mathrm{d} / \mathrm{d} v)^{m} A_{k}(\nu)$ and $D_{k}^{(m)}(\nu)=(\mathrm{d} / \mathrm{d} \nu)^{m} D_{k}(\nu)$ will be the polynomials of degrees $m$ and $m+1$, respectively. By implication, $A_{k}^{(m)}(\nu)$ and $D_{k}^{(m)}(\nu)$ will have $m$ and $m+1$ zeros, respectively. The same statement holds true for the resulting $\operatorname{Re}\left(G_{k}^{(m)}(v)\right)$ and $\operatorname{Im}\left(G_{k}^{(m)}(v)\right)$ obtained by subjecting (13) and (14) to the operator $(\mathrm{d} / \mathrm{d} \nu)^{m}$ in the case of the purely real amplitudes $d_{k}\left(\varphi_{k}=0,1 \leq k \leq K\right)$. As a consequence of these numerators' zeros, the real and imaginary derivative spectra $\operatorname{Re}\left(G_{k}^{(m)}(\nu)\right)$ and $\operatorname{Im}\left(G_{k}^{(m)}(v)\right)$ will have $m$ and $m+1$ lobes, respectively, symmetrically distributed around the resonance chemical shift, $\zeta_{k}$. The polarity of each given individual lobe will be switched from positive to negative when $m$ passes from an even $(m=2 l)$ to an odd $(m=2 l+1)$ number, where $l$ is a positive integer. This will be illustrated in the Results section.

\subsubsection{Magnitude modes of derivative spectra}

The appearance of multiple lobes (with the main lobes and side lobes) in the plots of real and imaginary derivative spectra would be an additional task for interpretation and metabolite assignments. Instead, it is more straightforward to analyze the corresponding magnitude mode $\left|G_{k}^{(m)}(v)\right|$ of complex spectrum $G_{k}^{(m)}(v)$ :

$$
\begin{aligned}
& g_{k, m}(v) \equiv\left|G_{k}^{(m)}(v)\right| \quad(\text { Magnitude: derivative spectrum) }, \\
& g_{k, m}(v)=\frac{\left|d_{k}\right|}{2 \pi} m !\left[\left(v-\zeta_{k}\right)^{2}+\lambda_{k}^{2}\right]^{-\frac{m+1}{2}} \quad(m=1,2, \ldots) .
\end{aligned}
$$

The peak height $h_{k, m}$ of the lineshape in the magnitude $m$ th derivative spectrum is deduced from (40) at $v=\zeta_{k}$ as:

$$
\begin{aligned}
& h_{k, m}=g_{k, m}\left(\zeta_{k}\right) \quad(\text { Peak height: Magnitude of the derivative spectrum }), \\
& \therefore h_{k, m}=\frac{m !}{2 \pi} \frac{\left|d_{k}\right|}{\lambda_{k}^{m+1}} \quad(m=1,2, \ldots) .
\end{aligned}
$$


Of course, the same result (52) is obtained by taking the absolute value of the complex rhs of (48) at $v=\zeta_{k}$, i.e. $h_{k, m}=\left|H_{k, m}\right|$. It is seen that the numerator of $h_{k, m}$ increases factorially, i.e. as $m$ ! when $m$ is augmented. Another $m-$ dependence of $h_{k, m}$ comes from its denominator $\lambda_{k}^{m+1}$. However, for reconstruction of the fundamental parameters from the magnitude of the $m$ th derivative spectrum, what matters in the end is their relations to the reference non-derivative $(m=0)$ absorption lineshape $A_{k}(v)$. The sought absorptive peak height $h_{k}$ from (35) and $h_{k, m}$ from (52) are related by:

$$
h_{k, m}=\frac{m !}{\lambda_{k}^{m}} h_{k}
$$

Thus, if we have $h_{k, m}$ and $\lambda_{k}$, the peak height $h_{k}$ of absorption can be deduced from (53). However, $\lambda_{k}$ is yet to be found. This can be done by connecting the breadth $\xi_{k}$ of absorption ( $m=0)$ from (8) to its counterpart for the magnitude of the $m$ th $(m>1)$ derivative spectrum.

To determine the lineshape breadth of the magnitude of the $m$ th derivative spectrum $g_{k, m}(v)$, it suffices to resort to the very definition of the full width at half maximum, FWHM. The lineshape of $g_{k, m}(v)$ is a single bell-shaped symmetric Lorentzian function. The FWHM of this profile is the distance between the two crossing points of the constant line $h_{k, m} / 2$ (the half maximum) and the Lorentzian $g_{k, m}(v)$ itself in a plot with $v$ as the abscissa. Prior to using this condition, inherent in the definition of the FWHM, we first rewrite (53) as follows:

$$
\frac{2 \pi}{\left|d_{k}\right| m !} g_{k, m}(v)=\left[\left(v-\zeta_{k}\right)^{2}+\lambda_{k}^{2}\right]^{-\frac{m+1}{2}},
$$

or equivalently,

$$
\left[\frac{\left|d_{k}\right| m !}{2 \pi g_{k, m}(v)}\right]^{\frac{2}{m+1}}=\left(v-\zeta_{k}\right)^{2}+\lambda_{k}^{2}
$$

It is here that we evoke the mentioned crossing of the constant line (constant in the sense of being independent of $v$ ). To place that line at the half maximum of $g_{k, m}(v)$, we set $v=\zeta_{k}$ on the lhs of (55) and divide the result $g_{k, m}\left(\zeta_{k}\right)=h_{k, m}$ by 2 to obtain $h_{k, m} / 2$ so that the condition for the FWHM reads as:

$$
\left[\frac{\left|d_{k}\right| m !}{2 \pi h_{k, m} / 2}\right]^{\frac{2}{m+1}}=\left(v-\zeta_{k}\right)^{2}+\lambda_{k}^{2} .
$$

Using (52), the lhs of (56) is reduced to $2^{2 /(m+1)} \lambda_{k}^{2} \equiv \beta_{k, m}^{2}$. With this latter quantity at hand, the algebraic form (55) becomes:

$$
\nu^{2}-2 \zeta_{k} \nu+\alpha_{k}=0, \quad \alpha_{k}=\zeta_{k}^{2}+\lambda_{k}^{2}-\beta_{k, m}^{2}, \quad \beta_{k, m}=2^{1 /(m+1)} \lambda_{k}
$$


The roots of the quadratic equation (57) are given by:

$$
v_{ \pm}^{\{k, m\}}=\zeta_{k} \pm \lambda_{k}\left[2^{2 /(m+1)}-1\right]^{1 / 2}
$$

The frequencies $v_{+}^{\{k, m\}}$ and $\nu_{-}^{\{k, m\}}$ are the positions of the crossings between the mentioned horizontal line drawn at the half of the maximum of the magnitude of the $m$ th derivative peak, $h_{k, m / 2}$, and the peak profile itself. The crossing points $v_{+}^{\{k, m\}}$ and $v_{-}^{\{k, m\}}$ are located to the right and to the left of the peak position $\zeta_{k}$, respectively. Their difference is the sought quantity $\{\mathrm{FWHM}\}_{k, m}$ of the magnitude mode of the $m$ th derivative of the $k$ th component peak. Thus, we have:

$$
\begin{aligned}
& \Delta v^{\{k, m\}} \equiv v_{+}^{\{k, m\}}-v_{-}^{\{k, m\}}=\{\mathrm{FWHM}\}_{k, m}, \\
& \therefore\{\mathrm{FWHM}\}_{k, m}=2 \lambda_{k}\left[2^{2 /(m+1)}-1\right]^{1 / 2} .
\end{aligned}
$$

Moreover, the half sum of $v_{+}^{\{k, m\}}$ and $v_{-}^{\{k, m\}}$ gives the peak position, $\zeta_{k}$ :

$$
\frac{1}{2}\left(v_{+}^{\{k, m\}}+v_{-}^{\{k, m\}}\right)=\zeta_{k}
$$

In (60), the term $2 \lambda_{k}$ is the $\{\mathrm{FWHM}\}_{k} \equiv \xi_{k}$ of absorption $A_{k}(v)$ according to (8). This gives the relationship between the full width at half maximum of the magnitude of the $m$ th derivative spectrum $\{\mathrm{FWHM}\}_{k, m}$ and that of the absorption $\{\mathrm{FWHM}\}_{k}$ :

$$
\begin{aligned}
& \{\mathrm{FWHM}\}_{k, m}=\left[2^{2 /(m+1)}-1\right]^{1 / 2}\{\mathrm{FWHM}\}_{k} \quad(m=0,1,2, \ldots), \\
& \{\mathrm{FWHM}\}_{k, m}<\{\mathrm{FWHM}\}_{k},\left[2^{2 /(m+1)}-1\right]^{1 / 2}<1 \quad(m=2,3,4, \ldots) .
\end{aligned}
$$

The result $(62 \mathrm{a})$ is for any $m(m=0,1,2, \ldots)$. In the special case for $m=0$ (non-derivative magnitude spectrum), it follows from (62) that $\{\mathrm{FWHM}\}_{k, 0}=$ $\sqrt{3}\{\mathrm{FWHM}\}_{k}$, which also reads as $\{\mathrm{FWHM}\}_{k, 0}^{\mathrm{Magn}}=\sqrt{3}\{\mathrm{FWHM}\}_{k}^{\text {Absorp }}$, in agreement with (40). Another particular case is $m=1$ (magnitude of the 1st derivative spectrum), for which (62) yields $\{\mathrm{FWHM}\}_{k, 1}=\{\mathrm{FWHM}\}_{k}$, or equivalently, $\{\mathrm{FWHM}\}_{k, 1}^{\mathrm{Magn}}=\{\mathrm{FWHM}\}_{k}^{\text {Absorp }}$. In other words, the full width at half maximum is the same in the absorption and the magnitude of the 1st derivative spectrum. For $m \geq 2$, according to (62b), the linebreadth of the magnitude of the $m$ th derivative of the $k$ th component spectrum is narrower than that of the corresponding non-derivative $(m=0)$ absorption profile. Therefore, such derivative spectra make the profile thinner and, hence, improve resolution. This circumstance simultaneously highlights the roles of both derivatives and linebreadths: the higher the derivative order, the thinner the profile width, and the better the resolution. It is seen from (62a) that relative to the non-derivative width $\{\mathrm{FWHM}\}_{k}$, the rate of narrowing of the linebreadth $\{\mathrm{FWHM}\}_{k, m}$ of the derivative spectrum decreases slowly with augmentation of the differentiation 
order $m$. This is implied by a very slow decrease of the exponent $2 /(m+1)$ of the number 2 in the multiplicative factor $\left[2^{2 /(m+1)}-1\right]^{1 / 2}$ from $(62 \mathrm{a})$. Such a circumstance implies that the higher order derivatives (larger $m$ ) are needed for substantial resolution improvement of derivative lineshapes, compared to their non-derivative counterparts.

\section{Exact fundamental parameters from high-order derivatives of total shape spectra}

Recently [28], we have used the dFPT to show that the lineshapes of the components can be reconstructed directly from non-parametrically computed total shape spectra or envelopes without solving the quantification problem. This was possible for highorder derivatives of envelopes whose magnitude modes were the most straightforward for interpretation. In Ref. [28], these computed envelopes were plotted to visualize and monitor the main features of the obtained magnitude derivative spectra. The most salient characteristics of the components of the magnitudes of the derivative envelopes $(m>0)$, relative to the non-derivative $(m=0)$ absorptions, as a function of the increased derivative order $m$, were the pronounced: (i) constancy of the peak positions, (ii) narrowing of the peak widths and (iii) augmentation of the peak heights. The cause of such properties was attributed in Ref. [28] to the significantly reduced or nearly eliminated interference effects among resonances in a given total shape spectrum or envelope. It is this suppressed interference which, for sufficiently high derivative order $m$, facilitated a clear visualization of the individual components that were completely hidden in some compound peaks from the given non-derivative $(m=0)$ envelope. Further, besides the stable peak positions, it is also important to have the peak heights stabilized. This can be achieved with peak height normalization by equalizing the heights of the same tallest peak in both the entire magnitude derivative envelope $\left|G^{(m)}(v)\right|$ for a fixed $m \geq 1$ and in the corresponding non-derivative absorption $(m=0)$ :

$$
\max \left\{\left|G^{(m)}(v)\right|\right\}=\max \left\{\operatorname{Re} G^{(0)}(v)\right\} \quad(m=1,2, \ldots),
$$

where $G^{(0)}(\nu)=G(\nu)$. It should be emphasized that this normalization is made only for the single, isolated tallest peak. If the reconstruction is truthful, all the other heights of the identified component peaks in the given envelope will also be correct. This was indeed the case in the dFPT from Ref. [28], where the input peak heights associated with synthesized time signals were recovered exactly. Note that $\max \{|G(v)|\}$ was used in Ref. [28], instead of the corresponding $\max \{\operatorname{Re} G(v)\}$ in the rhs of (63). This was justified since in the considered example with lactate (Lac) as the single, isolated tallest resonance, $\max \left\{\left|G^{(m)}(v)\right|\right\}(m>0)$ and $\max \{\operatorname{Re} G(v)\}(m=0)$ coincide.

All the mentioned features (i)-(iii), established computationally and visually observed in Ref. [28], have been theoretically confirmed in subsection 2.7.3. Moreover, in the current study, we proceed one critical step beyond Ref. [28]. This is to be accomplished by explicitly showing how subsection 2.7 .3 should be used to deduce the non-derivative $(m=0)$ absorption peak parameters from the extracted 
peak parameters of the magnitude of the derivative total shape spectrum $\left|G^{(m)}(v)\right|$ of a given differentiation order $m$. The provision for this demonstration is that the nonparametric FPT and the dFPT have converged relative to: (a) the model order $K$ and (b) the derivative order $m$ (regarding the positions and normalized heights) of all the identified components. This was the case with the present computations, the findings of which will be given in the Results section.

Another remark should also be made to clarify the connection of the findings from Ref. [28] with the present paper. Namely, in Ref. [28], the main goal was to show through exclusively non-parametric processing that the component spectra of a nonderivative absorption $(m=0)$ in the FPT can be extracted from the magnitude of the high-order derivative envelope in the dFPT. On the other hand, subsection 2.7.3 does not deal with the total shape spectra, i.e. envelopes. Rather, it explicitly deals with the magnitude $\left|G_{k}^{(m)}(v)\right|$ of the $m$ th derivative of the $k$ th component of the total shape spectrum $\left|G^{(m)}(v)\right|$ with $K$ resonances:

$$
\left|G^{(m)}(v)\right|=\left|\sum_{k=1}^{K} G_{k}^{(m)}(v)\right| .
$$

Moreover, by reference to (44), if (66) is to relate to subsection 2.7.3, it would explicitly read as:

$$
\left|G^{(m)}(v)\right|=\frac{m !}{2 \pi}\left|\sum_{k=1}^{K} \frac{d_{k}}{\left(v-v_{k}-i \lambda_{k}\right)^{m+1}}\right| .
$$

The rhs of (65) is the parametric representation of the envelope $\left|G^{(m)}(v)\right|$ for the known pairs $\left\{v_{k}, d_{k}\right\}$. However, the same fundamental pairs $\left\{v_{k}, d_{k}\right\}(k=1,2, \ldots, K)$ were treated as unknown in Ref. [28], where the task was to extract them by the nonparametrically computed $\left|G^{(m)}(v)\right|$ in the dFPT.

The stated remark is properly taken into account in the dFPT under the already formulated condition for the stabilization of peak positions and peak heights in the normalized amplitude mode of the $m$ th derivative envelopes for sufficiently high values of both $K$ and $m$. Namely, when the latter condition is fulfilled, it was seen in Ref. [28] that the non-parametrically computed envelope $\left|G^{(m)}(v)\right|$ correctly reconstructed all its components, such that the peak position and peak heights in the normalized total shape spectrum could clearly be seen to coincide with the corresponding input data. In Padé-based processing, the non-parametric estimation of $\left|G^{(m)}(v)\right|$ and its parametric version (65) yield the same component lineshapes for the non-derivative ( $m=0$, Ref. [15]) and derivative ( $m \geq 1$, present work) envelopes. Therefore, the spectral pairs extracted by the dFPT from the envelope $\left|G^{(m)}(v)\right|$ computed non-parametrically in Ref. [28] should coincide with the reconstructions that solve the quantification problem. Such an equivalence is facilitated by the analysis from subsection 2.7.3. Therein (and this is gist of the matter), the parametric dFPT is not used to generate the derivative spectra for practical purposes. Rather, such a parametric formalism is employed only to establish the exact theoretical relationships among the sought parameters $\left\{v_{k}, d_{k}\right\}$ 
of the non-derivative absorption $(m=0)$ and the magnitude of the $m$ th derivative of the $k$ th component spectrum. With the availability of these relations, we can return to the envelope $\left|G^{(m)}(v)\right|$ which is non-parametrically computed by the dFPT. This time, however, the relationships from subsection 2.7.3 are to be "inverted". Namely, by considering the resonance positions, widths and heights as the known quantities retrievable from the well-resolved $m$ th derivative envelope $\left|G^{(m)}(v)\right|$, we will determine the corresponding peak parameters $\left\{v_{k}, d_{k}\right\}$ of the non-derivative $(m=0)$ absorption lineshape. The reason for which the peak positions, widths and heights of the components from the mentioned non-parametrically computed derivative envelope can be considered as known, will be explicitly stated after giving the "inverse" relations among the parameters of the two kinds of spectra (derivative and non-derivative).

Importantly, the given high-order derivative envelope $\left|G^{(m)}(v)\right|$ from (64) practically annihilates all the interference effects among the components $G_{k}^{(m)}(v)$. Stated equivalently, these components become localized to such an extent that each of them behaves as if it were the only resonance in the entire Nyquist interval. With this feature at play, the analysis from subsection 2.7 .3 for $\left|G_{k}^{(m)}(v)\right|$ coherently relates to $G^{(m)}(v)$ in (64).

The outlined procedure runs as follows. First, formula (62) tells us that the sought $\{\mathrm{FWHM}\}_{k}$ of the absorption $A_{k}(v)$ from (15) can be obtained if $\{\mathrm{FWHM}\}_{k, m}$ for the magnitude spectrum is available for a fixed derivative order $m$. Therefore, from a bell-shaped Lorentzian lineshape $g_{k, m}(v)$ for the magnitude of the $m$ th derivative spectrum, the three parameters $\left\{\zeta_{k}, \lambda_{k},\left|d_{k}\right|\right\}$ of the absorption $A_{k}(v)$ in (15) can be extracted from the analytical expressions (35), (53) and (62), or explicitly, in their mentioned "inverse" forms:

$$
\begin{aligned}
\{\mathrm{FWHM}\}_{k} & =\left[2^{2 /(m+1)}-1\right]^{-1 / 2}\{\mathrm{FWHM}\}_{k, m} \\
\left|d_{k}\right| & =2 \pi \lambda_{k} h_{k} \\
\lambda_{k} & =\frac{1}{2}\{\mathrm{FWHM}\}_{k} \\
h_{k} & =\frac{\lambda_{k}^{m}}{m !} h_{k, m} .
\end{aligned}
$$

Thus, $\lambda_{k}$ as $\operatorname{Im}\left(v_{k}\right)$ and $\left|d_{k}\right|$ are obtainable as soon as $\{\mathrm{FWHM}\}_{k, m}$ and $h_{k, m}$ of the derivative spectrum are computed by the non-parametric dFPT on a very fine grid of the sweep frequencies $v$ (the finer, the better; this is possible since, as stated earlier, no preassigned Fourier grid frequencies are preconditioned in the FPT nor dFPT). The results for the spectra are used to plot the magnitude of the $m$ th derivative lineshape alongside which the same algorithm in the dFPT also provides the numerical values of $h_{k, m}, \zeta_{k}$ and $\{\mathrm{FWHM}\}_{k, m}$. The peak position $\zeta_{k}$ is the same in the absorption (nonderivative, $m=0$ ) and the magnitude of the $m$ th derivative spectrum. This position is available since the computed peak height $h_{k, m}$ is located at $v=\zeta_{k}$. Also, this latter quantity $\zeta_{k}$ is, according to (61), the chemical shift of the midpoint of the segment $\{\mathrm{FWHM}\}_{k, m}$ computed by the dFPT. Observe, that actually the key is to find the intersection points $v_{ \pm}^{\{k, m\}}$ that, in turn, provide both $\{\mathrm{FWHM}\}_{k, m}$ and $\zeta_{k}$, as per (59)- 
(61). This completes the reconstruction of the parameters $\left\{\zeta_{k}, \lambda_{k},\left|d_{k}\right|\right\}$ by the dFPT as a lineshape analyzer. Similarly, the phase $\varphi_{k}$ of $d_{k}$ in (6) can also be reconstructed from the computed complex derivative spectrum $G_{k}^{(m)}(v)$. The pertinent analytical expression from which $\varphi_{k}$ can be retrieved is not given in subsection 2.7 since the present Results section deals only with real $d_{k}$, i.e. $d_{k}=\left|d_{k}\right|\left(\varphi_{k}=0,1 \leq k \leq K\right)$.

\section{Results}

Here, we will present two sets of results in two subsections. Subsections 4.1 and 4.2 are on the para-diagonal and diagonal rational polynomials, respectively. These polynomial quotients are of the Padé forms that belong to the Lorentzian-type frequency distributions. Both subsections are primarily focused on the derivative envelopes. The derivative transforms $(\mathrm{d} / \mathrm{d} \nu)^{m}$ of high order $m$ are of the main interest and this is considered in subsection 4.2. The non-derivative $(m=0)$ envelopes to which the differentiation operator $(\mathrm{d} / \mathrm{d} \nu)^{m}$ with $m>0$ is applied will also be given. This is done with the purpose of monitoring the stabilization of the derivative spectra with increasing $m$ so as to establish their resolution capabilities relative to the reference absorptive envelopes for $m=0$. In subsection 4.1, the paradiagonal Padé approximants in their non-derivative $(m=0)$ and derivative ( $m=1$ and $m=2)$ versions are used to directly and quantitatively visualize the relationships among their input peak parameters (positions, widths, heights). Therein, it will also be shown how these latter peak parameters can be recovered from the computed spectrum using the analytical expressions listed in subsection 4.1. In subsection 4.2, the non-parametric Padé-based reconstructions as lineshape estimations, are employed to generate non-derivative and derivative envelope spectra.

\subsection{Direct and quantitative visualization of the peak positions, widths and heights in derivative versus non-derivative envelopes}

\subsubsection{Analytical expressions}

We herein analyze the non-derivative and derivative Lorentzians in different modes of spectral representations. For a visual quantification by way of reading off the peak parameters (positions, widths, heights) directly from the plotted graphs, we shall take a single absorptive $R_{\mathrm{A}}(v)$ and dispersive $R_{\mathrm{D}}(v)$ Lorentzian:

$$
\begin{aligned}
& R_{\mathrm{A}}(v)=d_{0} \frac{\Gamma_{0}^{2}}{\left(v-v_{0}\right)^{2}+\Gamma_{0}^{2}}, \\
& R_{\mathrm{D}}(v)=d_{0} \Gamma_{0} \frac{v-v_{0}}{\left(v-v_{0}\right)^{2}+\Gamma_{0}^{2}} .
\end{aligned}
$$

Absorption $R_{\mathrm{A}}(v)$ in (70) is of the type $Y_{\mathrm{A}}(v)$ from (3) with the same resonance frequency $v_{0}$, but with a different normalization. Further, the FWHM of the nonderivative absorption peak in (70) is equal to $2 \Gamma_{0}$, i.e. 
$\mathrm{FWHM}=2 \Gamma_{0}\left(\right.$ Full width at half maximum of absorption $\left.R_{\mathrm{A}}(v)\right)$.

For the analysis in this subsection, the 1st, 2nd and 3rd derivatives of $R_{\mathrm{A}}(v)$ from (70) will be considered with the same resonance frequency $v_{0}$ and the real amplitude $d_{0}$. They read as:

$$
\begin{aligned}
& \frac{\mathrm{d}}{\mathrm{d} \nu} R_{\mathrm{A}}(v) \equiv R_{A}^{(1)}(v)=-2 d_{0} \Gamma_{0}^{2} \frac{v-v_{0}}{\left[\left(v-v_{0}\right)^{2}+\Gamma_{0}^{2}\right]^{2}}, \\
& \frac{\mathrm{d}^{2}}{\mathrm{~d} v^{2}} R_{\mathrm{A}}(v) \equiv R_{A}^{(2)}(v)=2 d_{0} \Gamma_{0}^{2} \frac{3 v^{2}-6 v_{0} v+\left(3 v_{0}^{2}-\Gamma_{0}^{2}\right)}{\left[\left(v-v_{0}\right)^{2}+\Gamma_{0}^{2}\right]^{3}} \\
& \frac{\mathrm{d}^{3}}{\mathrm{~d} \nu^{3}} R_{\mathrm{A}}(v) \equiv R_{A}^{(3)}(v)=-24 d_{0} \Gamma_{0}^{2} \frac{\left(v-v_{0}\right)\left[v^{2}-2 v_{0} v+\left(v_{0}^{2}-\Gamma_{0}^{2}\right)\right]}{\left[\left(v-v_{0}\right)^{2}+\Gamma_{0}^{2}\right]^{4}} .
\end{aligned}
$$

The spectral zeros of these Padé approximants are the real parts of the numerator polynomials:

$$
\begin{aligned}
v & =v_{0} \quad\left(\text { One zero of } R_{A}^{(1)}(v)\right) \\
v_{ \pm} & =v_{0} \pm \frac{\Gamma_{0}}{\sqrt{3}} \quad\left(\text { Two zeros of } R_{A}^{(2)}(v)\right) \\
v & =v_{0}, v^{ \pm}=v_{0} \pm \Gamma_{0} \quad\left(\text { Three zeros of } R_{A}^{(3)}(v)\right) .
\end{aligned}
$$

The two extremal values of $R_{A}^{(1)}(v)$ are located at the two zeros $v_{ \pm}$of $R_{A}^{(2)}(v)$ as:

$$
R_{A}^{(1)}\left(v_{ \pm}\right)=\mp \frac{3 \sqrt{3}}{8} \quad \frac{d_{0}}{\Gamma_{0}}
$$

This provides the height-to-depth distance in $R_{A}^{(1)}$ :

$$
R_{A}^{(1)}\left(v_{-}\right)-R_{A}^{(1)}\left(v_{+}\right)=\frac{6 \sqrt{3}}{8} \frac{d_{0}}{\Gamma_{0}} \quad\left(\text { Peak-to-dip vertical distance in } R_{A}^{(1)}(v)\right),
$$

where $6 \sqrt{3} / 8 \approx 1.2990$. As such, the ratio $d_{0} / \Gamma_{0}$ becomes available from reading off the difference $R_{A}^{(1)}\left(v_{-}\right)-R_{A}^{(1)}\left(v_{+}\right)$in the plot of $R_{A}^{(1)}(v)$ versus $v$. The distance between the positions $v_{+}$and $v_{-}$of the maximum $R_{A}^{(1)}\left(v_{+}\right)$and the minimum $R_{A}^{(1)}\left(\nu_{-}\right)$, respectively, is:

$$
\begin{aligned}
\Delta v^{\{1\}} & \equiv v_{+}-v_{-}=\frac{2 \Gamma_{0}}{\sqrt{3}} \quad\left(\text { Peak-to-dip horizontal distance in } R_{A}^{(1)}(v)\right), \\
\therefore \Delta v^{\{1\}} & =\frac{\text { FWHM }}{\sqrt{3}},
\end{aligned}
$$


where FWHM is the full width at half maximum of the non-derivative absorption $R_{A}$ (v) from (70). Thus, the value $\Delta v^{\{1\}}$ which is read off from the plot of $R_{A}^{(1)}(v)$ versus chemical shift $v$ permits extraction of the sought breadth FWHM of absorption $R_{A}(v)$, via FWHM $=\sqrt{3} \Delta v^{(1)}$.

Further, the three extremal values of $R_{A}^{(2)}(v)$ are at the positions of the three zeros $v_{0}$ and $v^{ \pm}$of $R_{A}^{(3)}(v)$ via:

$$
\begin{aligned}
R_{A}^{(2)}\left(v_{0}\right) & =-\frac{2 d_{0}}{\Gamma_{0}^{2}} \quad\left(\text { Minimum of } R_{A}^{(2)}(v)\right), \\
R_{A}^{(2)}\left(v^{ \pm}\right) & =\frac{1}{2 \Gamma_{0}^{2}} \quad\left(\text { Maximae of } R_{A}^{(2)}(v)\right) .
\end{aligned}
$$

Therefore, (83) allows retrieval of the quotient $d_{0} / \Gamma_{0}^{2}$ from the read off value of $R_{A}^{(2)}\left(v_{0}\right)$ on the curve for $R_{A}^{(2)}(v)$ as a function of $v$. The difference between the locations $v^{+}$and $v^{-}$of the two peaks $R_{A}^{(2)}\left(v^{+}\right)$and $R_{A}^{(2)}\left(v^{-}\right)$, respectively, is:

$$
\Delta v^{\{2\}} \equiv v_{+}-v_{-}=2 \Gamma_{0}, \quad\left(\text { Peak-to-peak horizontal distance in } R_{A}^{(2)}(v)\right),
$$

or, by reference to (72):

$$
\Delta v^{\{2\}}=\text { FWHM. }
$$

Hence, the value $\Delta v^{\{2\}}$ which is read off from the graph of $R_{A}^{(2)}(v)$ versus $v$ yields directly the FWHM of absorption $R_{A}(v)$.

All the expressions (70)-(86) are for the general (numerically unspecified) fundamental real-valued parameters $\nu_{0}, \Gamma_{0}$ and $d_{0}$. For an easier follow-up in the pertinent graphical illustration, we set:

$$
v_{0}=1, \quad \Gamma_{0}=1, \quad d_{0}=1 \quad \text { (Exact input data). }
$$

\subsubsection{Graphical illustration}

Figure 1 shows the non-derivative $(m=0)$ absorption and dispersion modes on panel (a). Panel (b) compares the lineshapes of the absorption $(m=0)$ and its 1 st derivative $(m=1)$. Likewise, panel (c) compares the profile shapes of the absorption for $(m=0)$ and its 2 nd derivative $(m=2)$. The absorption shown in all three panels (a)-(c) is the reference spectrum. Here, the word "reference" is used to mean that the non-derivative $(m=0)$ absorption is the standard spectrum whose peak parameters are also sought from the dispersion $(m=0)$ and the derivatives $(m>1)$ of the absorption. In panel (a), the absorption peak parameters can be both transparently visualized and directly read off to verify the exact agreement with the input data. Therein, we can specifically read off the peak position as $\nu_{0}=0$, the FWHM as $2 \Gamma_{0}=2$ (which yields $\Gamma_{0}=1$ ) and the peak height via $R_{\mathrm{A}}\left(v_{0}\right)=R_{\mathrm{A}}(0)=1$. Thus, the extracted value $R_{\mathrm{A}}(0)$ 
The Breit-Wigner Resonance Formula \& Its Derivatives as the Specific Examples of the Pade Approximant Quantitative Visualization of Spectral Parameters from Absorption, Dispersion, First \& Second Derivatives

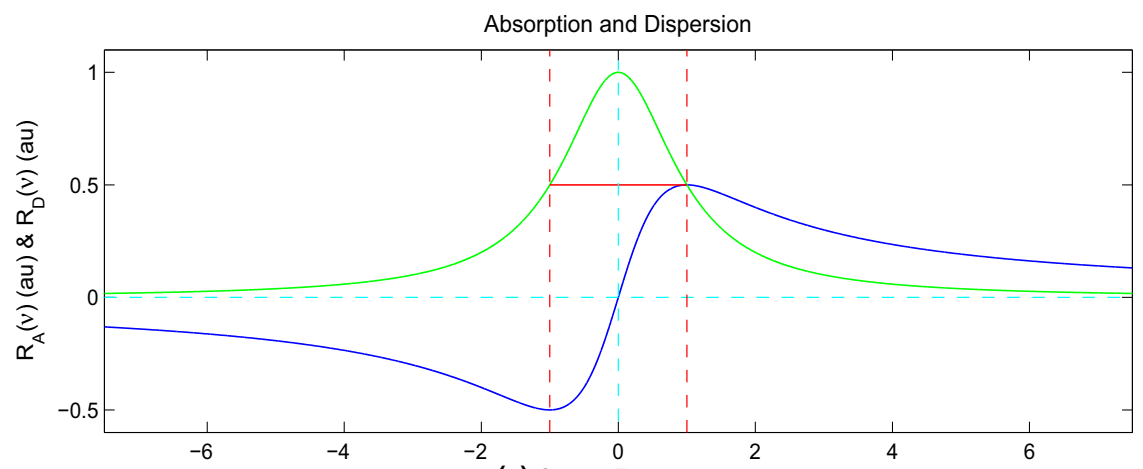

(a) Sweep Frequency $v$

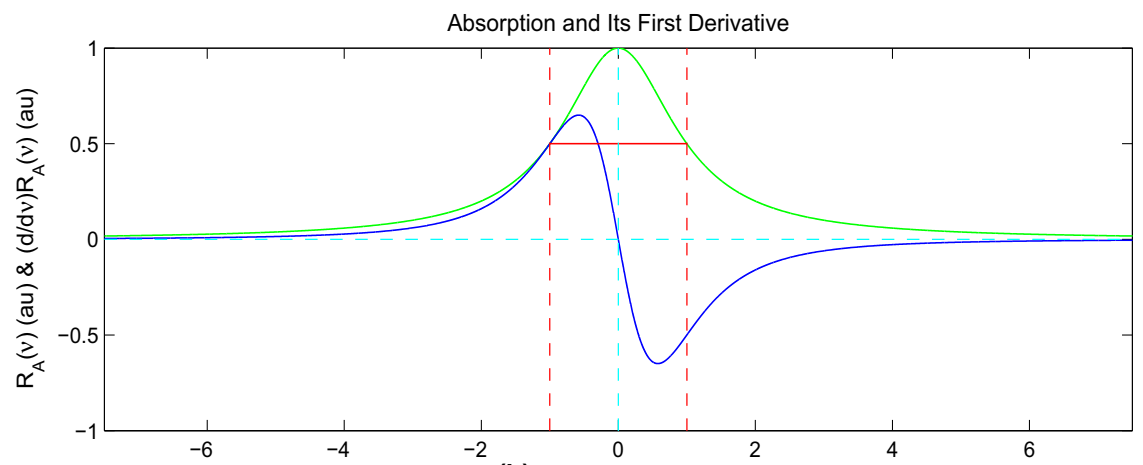

(b) Sweep Frequency $v$

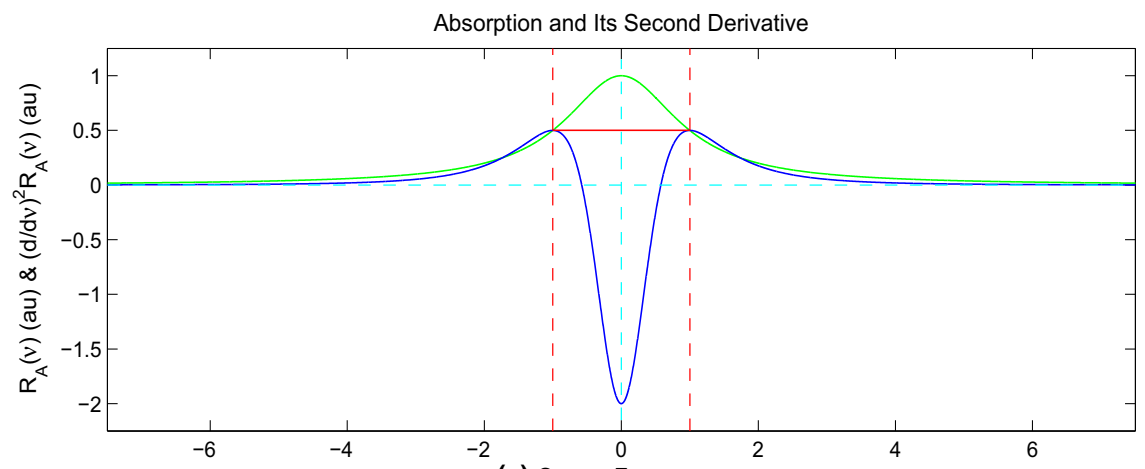

(c) Sweep Frequency $v$

Fig. 1 Quantitative visualization of the peak parameters from the non-derivative and derivative envelopes. (a) is for the non-derivative spectra $(m=0)$, whereas $(\mathbf{b}, \mathbf{c})$ additionally give the 1 st $(m=1)$ and 2nd $(m=2)$ derivative spectra, respectively. The non-derivative absorption and dispersion are computed from (70) and (71), whereas the 1st and 2nd derivative spectra are obtained from (73) and (74), respectively, with the input values: $v_{0}=0, \Gamma_{0}=1$ and $d_{0}=1$. (Color online) 
gives $d_{0}=1$, since, by definition, $R_{\mathrm{A}}\left(\nu_{0}\right)=d_{0} / \Gamma_{0}$. This completes the exact visual reconstructions of all the three peak parameters $\nu_{0}=0, \Gamma_{0}=1$ and $d_{0}=1$ for the non-derivative absorptive spectrum in (70). Crucially, however, such a retrieval need not be only visual. It can also be computational and, in fact, that is what needs to be done in practice with derivative spectra. To this end, $R_{\mathrm{A}}(v)$ is computed on a dense grid of the sweep frequencies $v$, so that $\max \left\{R_{\mathrm{A}}(v)\right\}$ is the exact peak height which is, by definition, located at $v_{0}$. Thus, the peak position and its height are both computed. The corresponding FWHM can also be computed by the same program. All that is needed is to simply extract the two intersecting points of $R_{\mathrm{A}}(v)$ with the line $\max \left\{R_{\mathrm{A}}(v)\right\} / 2$ parallel to the frequency abscissa at the half maximum. The positions of these two intersecting frequencies are located to the right and to the left of the resonance position $v_{0}$, respectively.

The same principle also applies to the dispersion mode $(m=0)$ seen in panel (a) of Fig. 1. The only distinction is in the interpretation, because the lineshape of dispersions and absorptions are different, as is clear from panel (a). Namely, the non-derivative dispersion $(m=0)$ has two spectral structures, a maximum and a minimum. The difference between the frequency locations of these two structures in the dispersion extremae is the same FWHM which is present in the absorption. Further, the peak height of the absorption can also be read off from the dispersion as the difference $\max \left\{R_{\mathrm{D}}\left(v_{\max }\right)-\min \left\{R_{\mathrm{D}}\left(v_{\min }\right)\right\}=d_{0} / \Gamma_{0}\right.$. Here, $v_{\min }$ and $v_{\max }$ are the frequencies at which $R_{\mathrm{D}}(v)$ exhibits its minimum and maximum, respectively. Moreover, the absorptive resonance frequency $v_{0}$ is the halved sum of $v_{\max }$ and $v_{\min }$ in the dispersion. Further, the extracted $\Gamma_{0}$ and $d_{0} / \Gamma_{0}$ give $d_{0}$. Hence, all the absorptive peak parameters $\left(v_{0}, \Gamma_{0}, d_{0}\right)$ are also extractable from the dispersion lineshape. This is natural since the same information is present in both the absorption and dispersion. Generally, absorptions and dispersions are two entirely equivalent representations of the complex mode of a given spectrum. If, e.g. absorption is available, dispersion can uniquely be obtained and vice versa. This is the content of the Kroning-Kramers relationships that are, from the signal processing standpoint equal to the Hilbert transform [1].

The outlined procedure of reconstructing the peak parameters by shape estimation alone is not limited to the non-derivative $(m=0)$ spectra. This is respectively illustrated in panels (b) and (c) of Fig. 1 for the 1 st $(m=1)$ and 2 nd $(m=2)$ derivatives of the absorption spectrum $R_{\mathrm{A}}(v)$ from (70). The lineshape of the 1 st derivative spectrum (73) on panel (b) is qualitatively reminiscent of the dispersion mode on panel (a). However, the polarities of the lineshapes for $m=1$ in panel (b) and for $m=0$ (dispersion) in panel (a) are different (i.e. the minimum becomes a maximum, and vice versa). Also, as per (80) and (81), the maximum-to-minimum (peak-to-dip) distance in $R_{A}^{(1)}(v)$ panel (b) is different from that in $R_{\mathrm{D}}(v)$ on panel (a) of Fig. 1. Nevertheless, this is not an obstacle to the extraction of the correct quantities $\nu_{0}, \Gamma_{0}$ and $d_{0}$ of absorption $(m=0)$ from the 1 st derivative spectrum $R_{A}^{(1)}(v)$, as long as the proper relationships exist connecting the parameters of the pertinent lineshapes in panels (a) and (b). Precisely such relations are presented in subsection 4.1.1. Overall, the real part of the 1st derivative envelope permits exact identification of the non-derivative $(m=0)$ absorptive peak parameters $v_{0}, \Gamma_{0}$ and $d_{0}$. The same holds true for the imaginary part of the 1 st derivative envelope (not shown to avoid clutter). 
Panel (c) of Fig. 1 compares the real part of the 2 nd $(m=2)$ derivative spectrum with the absorptive ( $m=0$ ) peak. It is observed that the curve for $m=2$ exhibits a minimum and two maximae as the side lobes. The distance between the locations of the two maximae for $m=2$ is equal to the FWHM of the absorptive $(m=0)$ peak, i.e. it is $2 \Gamma_{0}$. The midpoint of this distance for $m=2$ gives the peak location $v_{0}$ of the absorption peak. Moreover, the dip in the 2 nd derivative envelope from panel (c) allows determination of the amplitude $d_{0}$ of the absorption $(m=0)$ peak. Namely, the analytical expression for $\left(\mathrm{d}^{2} / \mathrm{d} v^{2}\right) R_{\mathrm{A}}(\nu)$ gives $\min \left\{\left(\mathrm{d}^{2} / \mathrm{d} v^{2}\right) R_{\mathrm{A}}(v)\right\}=$ $-2 d_{0} / \Gamma_{0}^{2}$, as per (83). On the other hand, the dip is read off from panel (c) of Fig. 1 as $\min \left\{\left(\mathrm{d}^{2} / \mathrm{d} v^{2}\right) R_{\mathrm{A}}(v)\right\}=-2$. Thus, $-2 d_{0} / \Gamma_{0}^{2}=-2$ which gives $d_{0} / \Gamma_{0}^{2}=1$ and, hence, $d_{0}=1$ since according to (85) and (86), $\Gamma_{0}$ is directly read off as FWHM/2, which yields $\Gamma_{0}=1$. Thus, the lineshape of $\operatorname{Re}\left((\mathrm{d} / \mathrm{d} v)^{2} R_{\mathrm{A}}(v)\right)$ for $m=2$ allows the exact extraction of the three input peak parameters of $R_{\mathrm{A}}(\nu)$ for $m=0$ as $\nu_{0}=0$, $\Gamma_{0}=1$ and $d_{0}=1$.

All these geometrical presentations of the resonance signatures are given here with the purpose of illustrating what the computer algorithm for non-parametric processing actually produces and provides as the linelist of spectral parameters. Importantly, this computation is based only upon the given non-derivative $(m=0)$ and derivative $(m \geq 1)$ envelopes. In other words, the illustrations in Fig. 1 and the accompanying discussion demonstrate how, in principle, the non-derivative $(m=0)$ numerically exact absorptive peak parameters can be reconstructed from the real parts of the 1st $(m=1)$ and the 2 nd $(m=2)$ derivatives of the absorptive Lorentzian in (70). Overall, to reemphasize, the entire procedure is lineshape processing which yields the exactly reconstructed parameters. Namely, regarding subsection 4.1.1, once the input parameters $v_{0}, \Gamma_{0}$ and $d_{0}$ are specified, the corresponding spectra (70) and (71), as well as the 1 st and the 2 nd derivatives of $R_{\mathrm{A}}(v)$ are sampled. Subsequently, the ensuing lineshapes are used in the role of the input data as if the peak parameters $v_{0}, \Gamma_{0}$ and $d_{0}$ were never known, and, thus, are the subject of reconstructions. That is what is illustrated in this subsection by the quantitative visualization of the spectral resonance fingerprints associated with (70) and (71) for $m=0$, as well as with (73) and (74) for the $m$ th derivatives ( $m=1, m=2$ ) of $R_{\mathrm{A}}(v)$, respectively. The accuracy of the retrieved spectral parameters depends on the fine grid of the sweep frequencies for the reason that no quantification problem is solved (i.e. no eigen-problem, nor secular equations are tackled). In other words, to precisely compute the needed extremal values of e.g. $(\mathrm{d} / \mathrm{d} v)^{m} R_{\mathrm{A}}(v)$ with $m \geq 1$ from the corresponding input spectrum $R_{\mathrm{A}}(v)$ for $m=0$, the sampling of the running frequency $v$ must be sufficiently dense. In practice, within e.g. MRS, it is not the spectra $R_{\mathrm{A}}(v)$ or $R_{\mathrm{D}}(v)$ that are acquired by encoding. Rather, the time signals are measured and they are subsequently processed to reconstruct the complex spectral envelopes whose real and imaginary parts $R_{\mathrm{A}}(v)$ and $R_{\mathrm{D}}(v)$ are (70) and (71), respectively.

If signal processing is carried out by the derivative fast Fourier transform (dFFT), the coarse Fourier grid for sampling via $v_{k}^{F}(0 \leq k \leq N-1)$ for the sweep frequency $v$ is not appropriate for the outlined procedure. By contrast, when signal processing is performed with the dFPT, the reconstructed spectra are given by the analytical expressions that can be evaluated at any desired density of the frequency grid. Such a 
circumstance offers the possibility to compute $(\mathrm{d} / \mathrm{d} v)^{m} R(\nu)$ for $m \geq 0$ with maximal precision. This, in turn, guarantees the accurate extraction of the spectral parameters for the sought non-derivative $(m=0)$ absorptive peaks using the corresponding Padécomputed derivative total shape spectra $(\mathrm{d} / \mathrm{d} v)^{m} R(v)$ for $m>0$. Moreover, there is another, most important difference between the dFFT and dFPT. This concerns noise. It has been shown in Ref. [28] that the dFPT suppresses noise, whereas the dFFT amplifies noise. This latter amplification could be somewhat mitigated by apodization, e.g. through multiplication of the FID by the product of an exponential and the Heaviside step function. This is, however, not recommended because such a procedure would modify the input FID. By contrast, the dFPT uses the raw, unedited FID data, as the proper starting point to signal processing.

\subsection{Shape processing of time signals by the non-parametric derivative fast Padé transform, dFPT}

Here, we extend the procedure from subsection 4.1 to the problem of processing the given synthesized noiseless time signals. The processing method used will be the dFPT. The fundamental parameters $\left\{v_{k}, d_{k}\right\}$ from (4) in the input time signal are chosen to closely represent those reported in Ref. [29] using in vitro MRS to encode FIDs from human breast cancer tissue, excised at surgery. In Ref. [29], a high magnetic field strength $B_{0}=14.1$ tesla $(\mathrm{T})$ is used and the associated Larmor precession frequency $v_{L}$ is $v_{L}=600$ megahertz (MHz). This sets the relations for the sweep $v$ and fundamental $v_{k}$ frequencies in dimensionless units of parts per million $(\mathrm{ppm})$ via:

$$
\begin{aligned}
v(\mathrm{ppm}) & =v_{\mathrm{H}_{2} \mathrm{O}}(\mathrm{ppm})-\frac{v(\mathrm{~Hz})}{v_{\mathrm{L}}(\mathrm{Hz})}, \\
\operatorname{Re}\left(v_{k}\right)(\mathrm{ppm}) & =v_{\mathrm{H}_{2} \mathrm{O}}(\mathrm{ppm})-\frac{\operatorname{Re}\left(v_{k}\right)(\mathrm{Hz})}{v_{\mathrm{L}}(\mathrm{Hz})}, \\
\operatorname{Im}\left(v_{k}\right)(\mathrm{ppm}) & =\frac{\operatorname{Im}\left(v_{k}\right)(\mathrm{Hz})}{v_{\mathrm{L}}(\mathrm{Hz})},
\end{aligned}
$$

where $v_{\mathrm{H}_{2} \mathrm{O}}$ is the resonance frequency of water (taken here as $v_{\mathrm{H}_{2} \mathrm{O}}=4.68 \mathrm{ppm}$ ). The fundamental resonance parameters $\left\{v_{k}, d_{k}\right\}$ of 9 absorptive peaks $(m=0)$ in concordance with Ref. [29], are given by:

$$
\begin{aligned}
\mathrm{M}_{k}= & \{\mathrm{Lac}, \mathrm{Ala}, \mathrm{Cho}, \mathrm{PC}, \mathrm{PE}, \mathrm{GPC}, \beta-\mathrm{Glc}, \mathrm{Tau}, \mathrm{m}-\mathrm{Ins}\}, \\
\operatorname{Re}\left(v_{k}\right)= & \{1.332,1.471,3.212,3.220,3.221,3.232,3.251,3.273,3.281\} \mathrm{ppm}, \\
\operatorname{Im}\left(v_{k}\right)= & 0.0008 \mathrm{ppm}(1 \leq k \leq K) ; \operatorname{Im}\left(v_{k}\right)>0, \\
h_{k}= & \{691.150,62.832,7.854,23.562,176.715,17.671,56.941, \\
& 219.911,70.686\} \mathrm{au}, \\
\left|d_{k}\right|= & \{0.325,0.032,0.004,0.012,0.090,0.009,0.029,0.012,0.036\} \mathrm{au}, \\
\varphi_{k}= & 0 \operatorname{rad}(1 \leq k \leq K),
\end{aligned}
$$

where $\mathbf{M}_{k}$ is the $k$ th metabolite, $K=9, d_{k}=\left|d_{k}\right| \mathrm{e}^{i \varphi_{k}}$ and $\varphi_{k}$ is the amplitude phase, presently taken as $\varphi_{k}=0 \mathrm{rad}(1 \leq k \leq 9)$ so that $d_{k}$ is real $\left(d_{k}=\left|d_{k}\right|\right)$ and $h_{k}$ is 
the $k$ th non-derivative $(m=0)$ peak height. Further, au denote arbitrary units. The bandwidth, BW, in Ref. [29] was $\mathrm{BW}=6000 \mathrm{~Hz}$, so that the sampling rate $\tau$ for the encoded complex signal is $\tau=1 / \mathrm{BW}=(1 / 6000) \mathrm{s}$. The total signal length in Ref. [29] was chosen to be extremely long (65536 sampled data points). This was preconditioned by the FFT which was used in Ref. [29] in the attempt to split apart the two closest resonances, phosphocholine (PC) at $3.220 \mathrm{ppm}$ and phosphoethanolamine (PE) at $3.221 \mathrm{ppm}$ separated by a very small chemical shift $(0.001 \mathrm{ppm})$. This excessiveness of the long encoded FIDs is unnecessary in Padé-based processing for which it suffices to use much shorter time signals. As such, we select the total length $N$ of the synthesized time signal to be $N=2048$ which is by a factor of 32 shorter than the 65536 data vector from Ref. [29]. The model order $K$ is set to be one half of $N$, i.e. $K=1024$. It is verified that all the Padé-reconstructed data (spectral envelopes and the extracted resonance parameters) have fully converged for $N=2048$ and $K=N / 2$. The same results (coincident for the non-parametric and parametric FPT and dFPT) are obtained for the signal length 4096 and the model order 2048. In this section, model order $K$ refers to the common degrees of the polynomials in the diagonal fast Padé transform, $P_{K}^{-}\left(z^{-1}\right) / Q_{K}^{-}\left(z^{-1}\right)$. The computations will be illustrated for the Padé variant $P_{K}^{-}\left(z^{-1}\right) / Q_{K}^{-}\left(z^{-1}\right)$, i.e. the $\mathrm{FPT}^{(-)}$and for its derivative version $\mathrm{dFPT}^{(-)}$, through $(\mathrm{d} / \mathrm{d} v)^{m} P_{K}^{-}\left(z^{-1}\right) / Q_{K}^{-}\left(z^{-1}\right)$, where the differentiation order $m$ is systematically increased $(m=1,2, \ldots, 50)$. The independent variable in these Padé response functions is the harmonic variable $z^{-1}$ given by $z^{-1}=\exp (-2 \pi i v \tau)$. Computations can be carried out with the same conclusions using any member of the Padé table $P_{L}^{-}\left(z^{-1}\right) / Q_{K}^{-}\left(z^{-1}\right)$ or $P_{L}^{-}(v) / Q_{K}^{-}(v)$, provided that convergence has been secured with respect to increasing polynomial degrees $L$ and $K$. This is the case in both subsections 4.1 and 4.2, where the para-diagonal $(L=K-1)$ and diagonal $(L=K)$ are used, respectively.

The results of the reconstruction by the $\mathrm{FPT}^{(-)}$and $\mathrm{dFPT}^{(-)}$for $m=0$ and $m \geq 1$, respectively, are presented in Figs. 2-9. The overall goal is to make in evidence that the basic features provided by subsection 4.1 for low derivative orders ( $m=1$ and $m=2$ ) are also present for any other value of the positive integer $m$. The reason for considering high derivative orders $m$ all the way up to $m=50$ in these illustrations is to establish confidence in reconstruction stability and in the unequivocal separation of the tightly overlapped PC and PE resonances. Of particular importance for tumor diagnostics is to unambiguously identify the PC peak and accurately extract its parameters from which the assigned metabolite concentrations can be determined. This is important in view of the fact that PC is a recognized cancer biomarker [30-32]. Information about the abundance of this metabolite in the scanned malignant tissue relative to the corresponding exceedingly minimal standard level in the healthy part of the same organ can be invaluable for early tumor diagnosis. This, in turn, could facilitate treatment management and enhance the chance for tumor control and potential cure of the patient.

The presentation of the illustrations is organized in a way which first shows only the pattern of the real parts of spectral derivative envelopes for varying differentiation order $m$ (Figs. 2 and 3). This is followed by comparisons of the lineshapes for the real and imaginary parts of the derivative envelopes (Figs. 4-6). Finally, the modes for the real parts and the absolute values (magnitudes) of the $m$ th derivative complex 
Nonparametric Quantification: dMRS, Synthesized Noiseless FIDs, Input Peak Heights (Filled Circles)

Real Parts of Envelopes: Derivative Fast Pade Transform, $\operatorname{Re}\left((\mathrm{d} / \mathrm{d} v)^{\mathrm{m}} \mathrm{P}_{\mathrm{K}}^{-} / \mathrm{Q}_{\mathrm{K}}^{-}\right) ; \mathrm{m}=0,4,8,12,16,20$

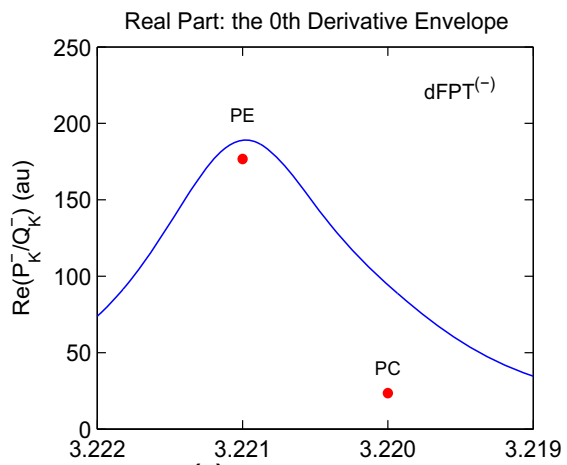

(a) Chemical Shift (ppm)

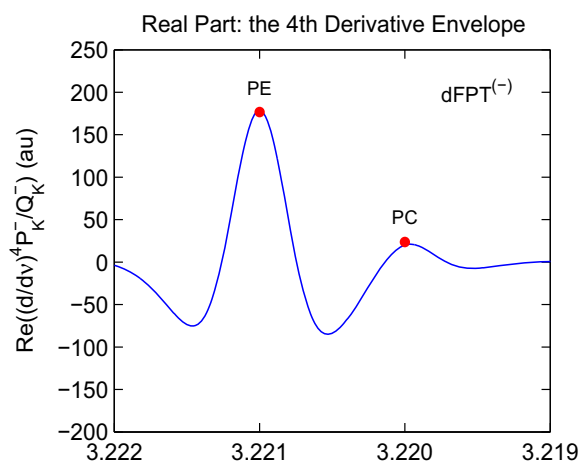

(b) Chemical Shift (ppm)

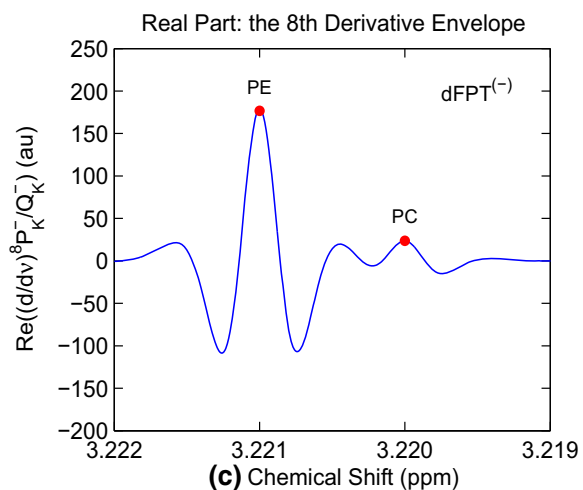

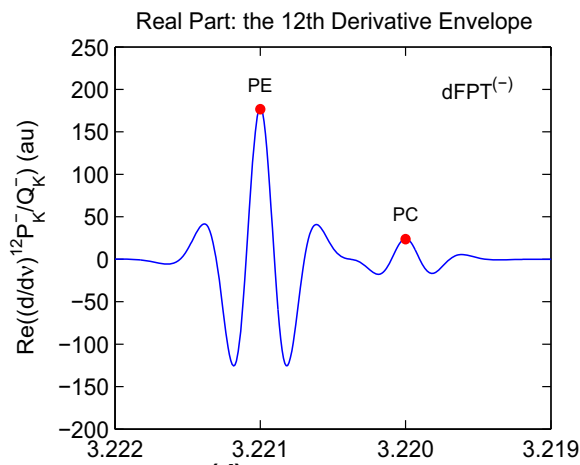

(d) Chemical Shift (ppm)

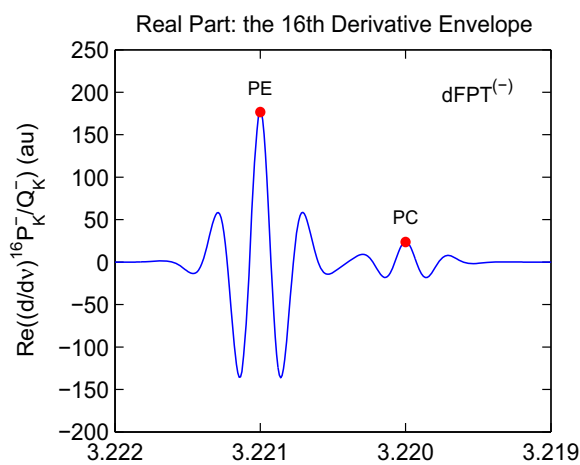

(e) Chemical Shift (ppm)

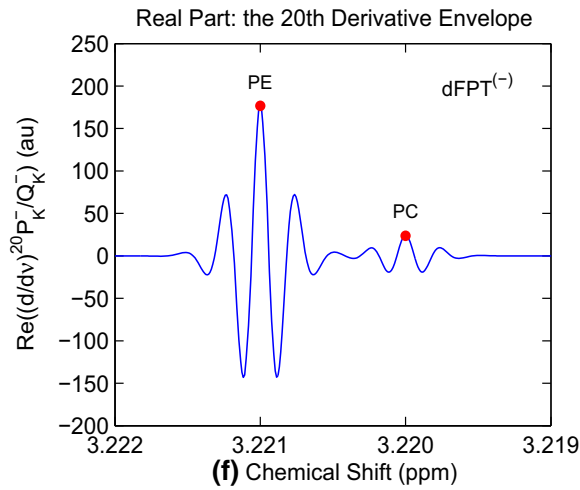

Fig. 2 Derivative fast Padé transform, $\mathrm{dFPT}^{(-)}$, for a synthesized time signal sampled by using $c_{n}$ from (4) and the spectral parameters (80) according to the FIDs encoded by in vitro MRS from excised human breast cancer tissue, as per Ref. [29]. (a) is for the non-derivative ( $m=0)$ absorption, whereas (b-f) are for the real part of the $m$ th derivative complex spectrum, where $m$ varies from 4 to 20 . Filled circles are the input peak heights $h_{k}$. (Color online) 
Nonparametric Quantification: dMRS, Synthesized Noiseless FIDs, Input Peak Heights (Filled Circles)

Real Parts of Envelopes: Derivative Fast Pade Transform, $\operatorname{Re}\left((\mathrm{d} / \mathrm{d} v)^{\mathrm{m}} \mathrm{P}_{\mathrm{K}}^{-} / \mathrm{Q}_{\mathrm{K}}^{-}\right) ; \mathrm{m}=0,24,28,32,36,40$

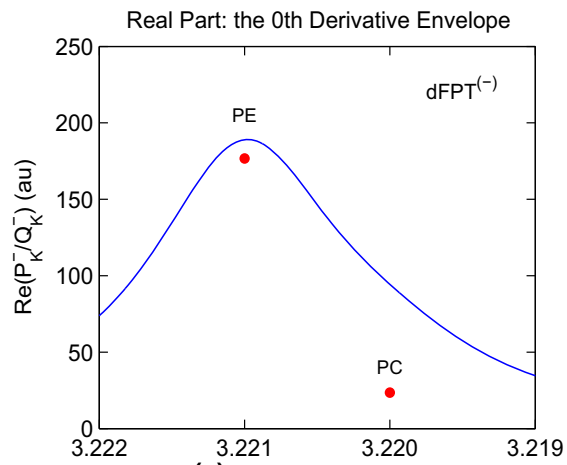

(a) Chemical Shift (ppm)

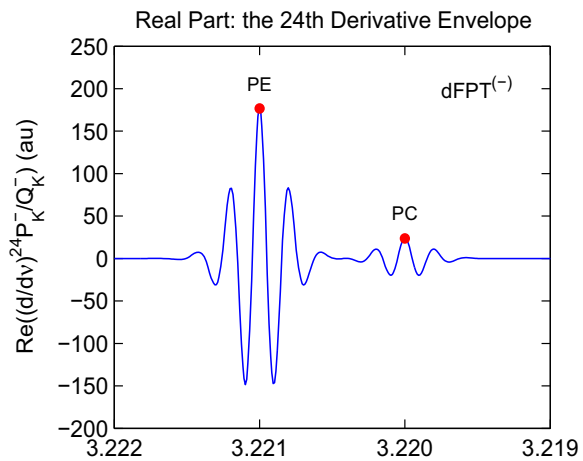

(b) Chemical Shift (ppm)

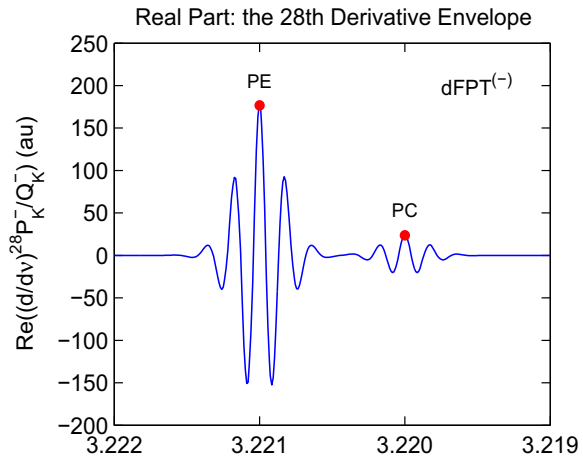

(c) Chemical Shift (ppm)

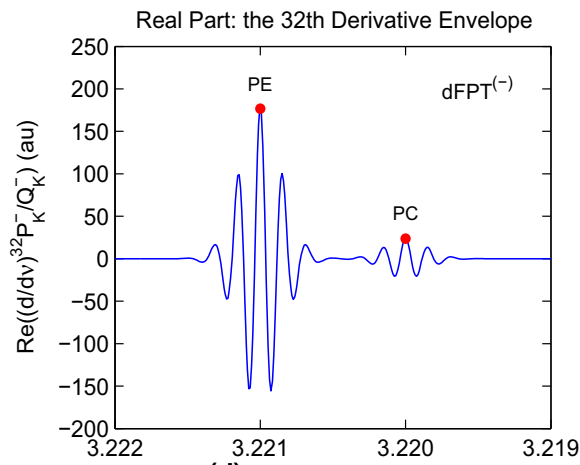

(d) Chemical Shift (ppm)

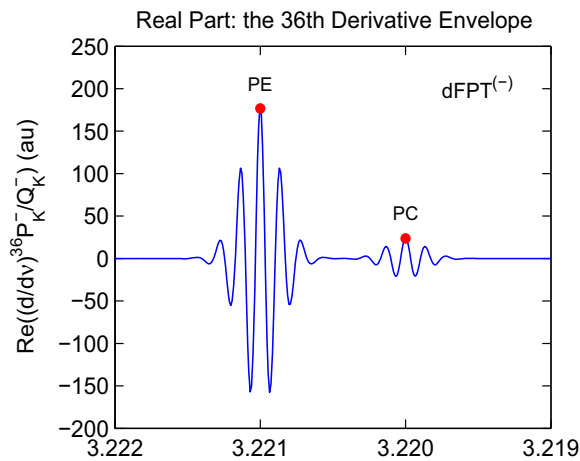

(e) Chemical Shift (ppm)

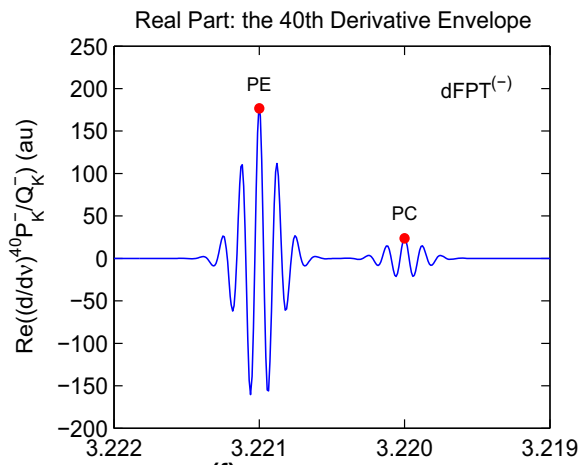

(f) Chemical Shift (ppm)

Fig. 3 Derivative fast Padé transform, $\mathrm{dFPT}^{(-)}$, for a synthesized time signal sampled by using $c_{n}$ from (4) and the spectral parameters (80) according to the FIDs encoded by in vitro MRS from excised human breast cancer tissue, as per Ref. [29]. (a) is for the non-derivative ( $m=0)$ absorption, whereas $(\mathbf{b}-\mathbf{f})$ are for the real part of the $m$ th derivative complex spectrum, where $m$ varies from 24 to 40 . Filled circles are the input peak heights $h_{k}$. (Color online) 
Nonparametric Quantification: dMRS, Synthesized Noiseless FIDs, Input Peak Heights (Filled Circles)

Real $(m=0,8,16)$ and Imaginary $(m=0,7,15)$ Parts of Pade Envelope Derivatives $(d / d v)^{m} P_{K}^{-} / Q_{K}^{-}$

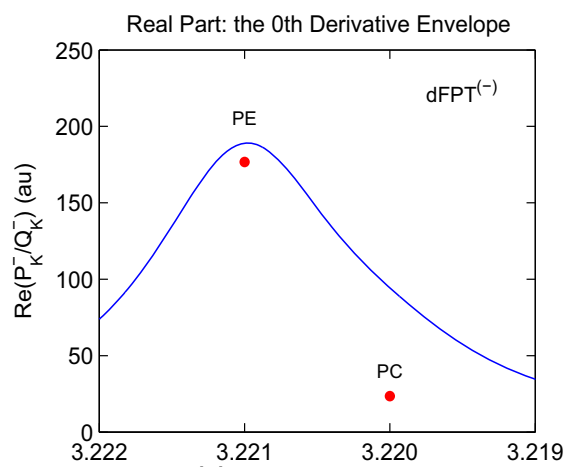

(a) Chemical Shift (ppm)

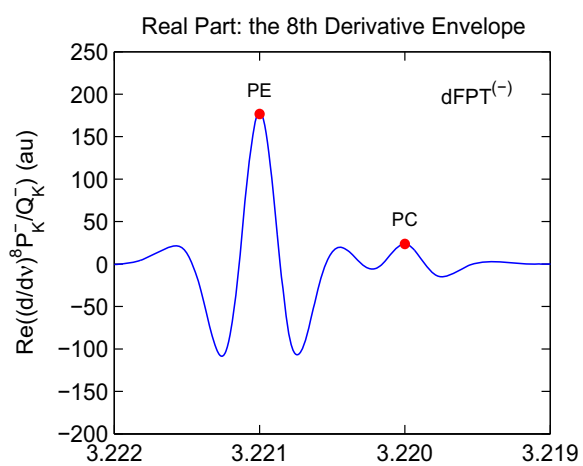

(b) Chemical Shift (ppm)

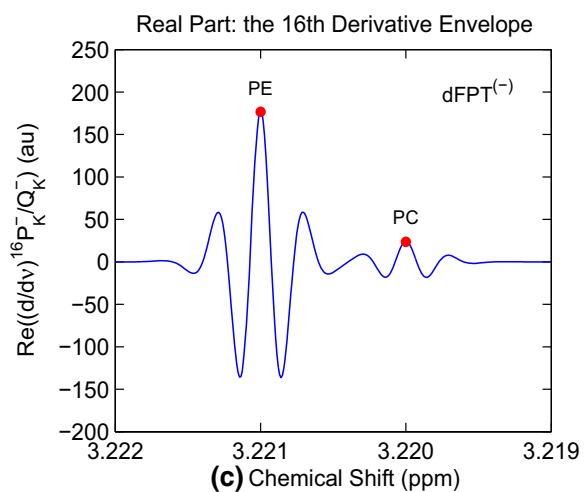

Imaginary Part: the Oth Derivative Envelope

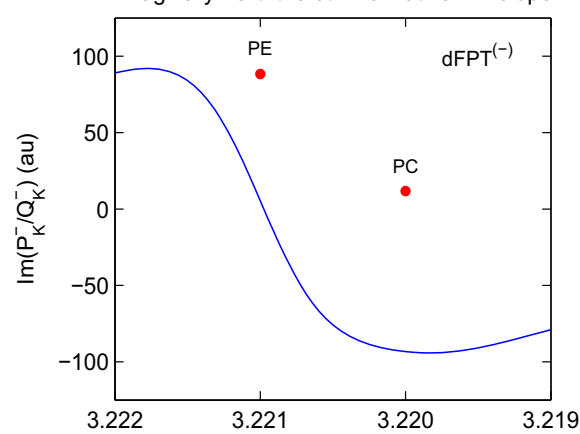

(d) Chemical Shift (ppm)

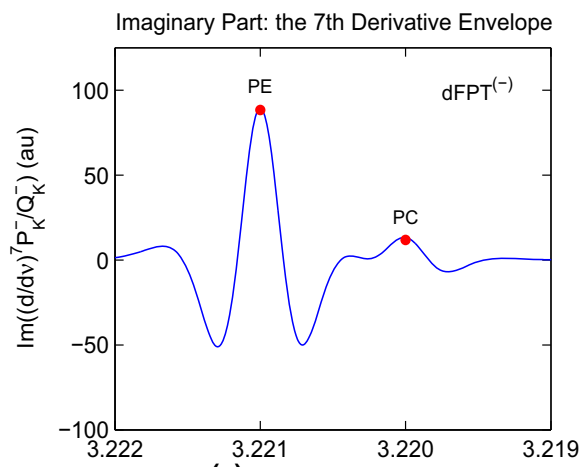

(e) Chemical Shift (ppm)

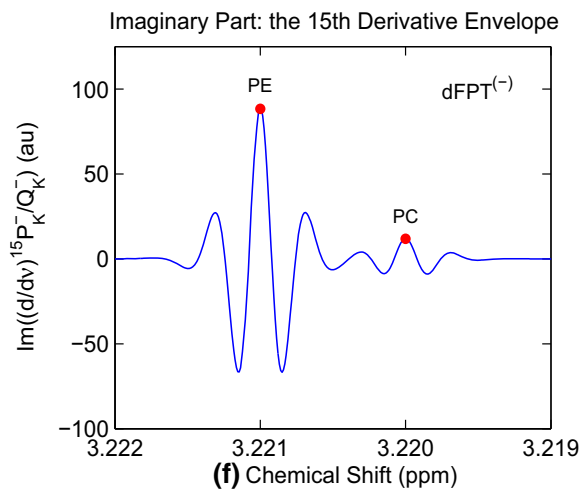

Fig. 4 Derivative fast Padé transform, $\mathrm{dFPT}^{(-)}$, for a synthesized time signal sampled by using $c_{n}$ from (4) and the spectral parameters (80) according to the FIDs encoded by in vitro MRS from excised human breast cancer tissue, as per Ref. [29]. The left and right columns are respectively for the real $(m=0,8,16)$ and imaginary $(m=0,7,15)$ parts of complex non-derivative and derivative envelopes. Filled circles are the input peak heights $h_{k}$. (Color online) 
Nonparametric Quantification: dMRS, Synthesized Noiseless FIDs, Input Peak Heights (Filled Circles) Real $(m=0,24,32)$ and Imaginary $(m=0,23,31)$ Parts of Pade Envelope Derivatives $(d / d v)^{m} P_{K}^{-} / Q_{K}^{-}$

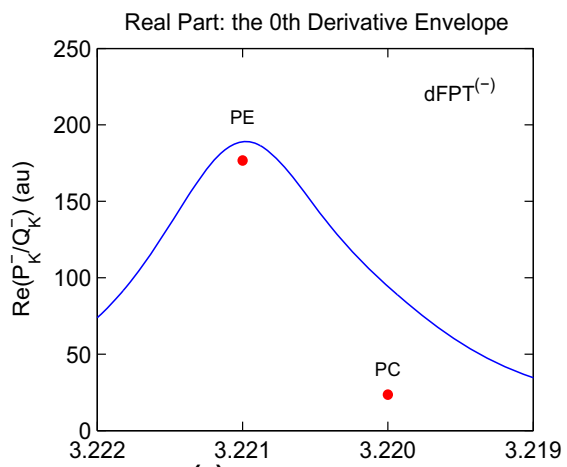

(a) Chemical Shift (ppm)

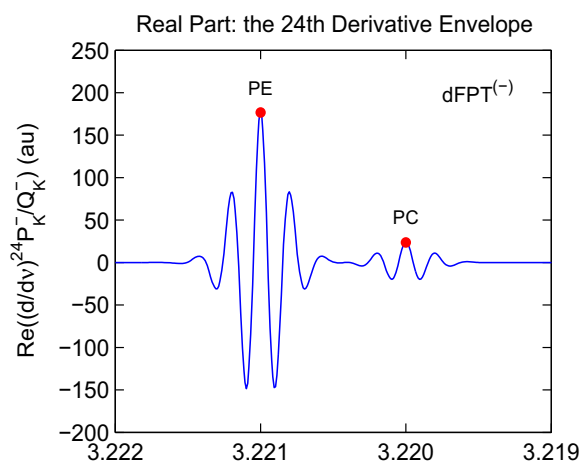

(b) Chemical Shift (ppm)

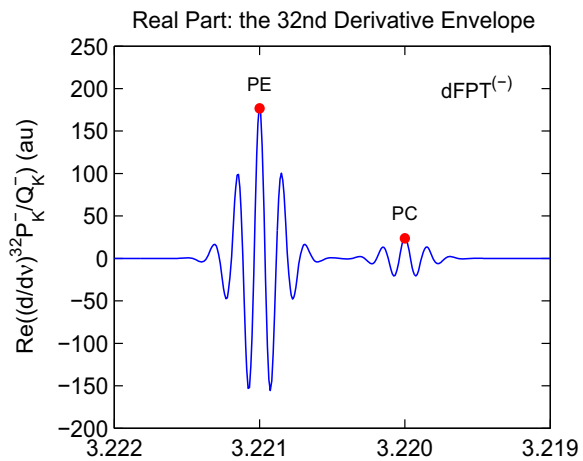

(c) Chemical Shift (ppm)

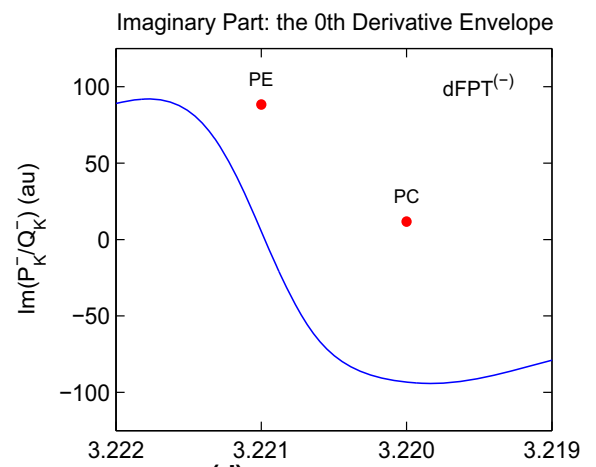

(d) Chemical Shift (ppm)

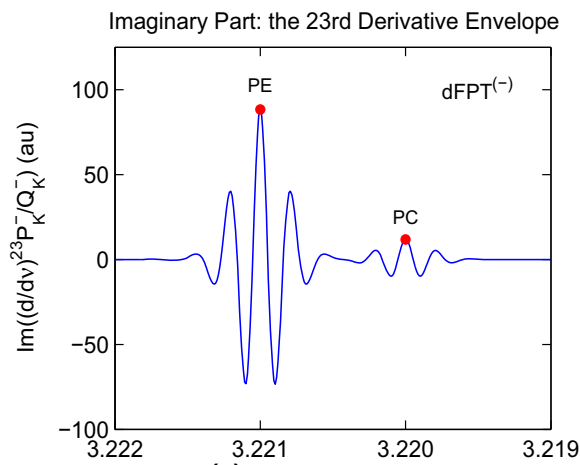

(e) Chemical Shift (ppm)

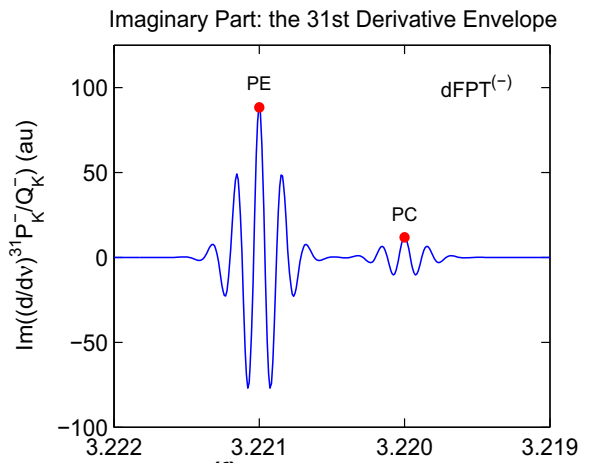

(f) Chemical Shift (ppm)

Fig. 5 Derivative fast Padé transform, $\mathrm{dFPT}^{(-)}$, for a synthesized time signal sampled by using $c_{n}$ from (4) and the spectral parameters (80) according to the FIDs encoded by in vitro MRS from excised human breast cancer tissue, as per Ref. [29]. The left and right columns are respectively for the real $(m=0,24,32)$ and imaginary $(m=0,23,31)$ parts of complex non-derivative and derivative envelopes. Filled circles are the input peak heights $h_{k}$. (Color online) 
Nonparametric Quantification: dMRS, Synthesized Noiseless FIDs, Input Peak Heights (Filled Circles) Real $(m=0,40,48)$ and Imaginary $(m=0,39,47)$ Parts of Pade Envelope Derivatives $(d / d v)^{m} P_{K}^{-} / Q_{K}^{-}$

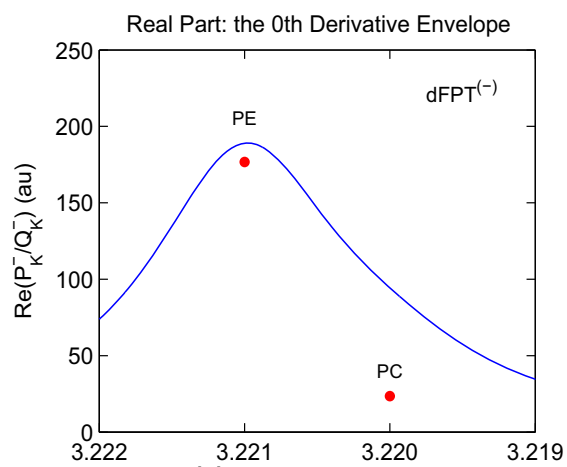

(a) Chemical Shift (ppm)

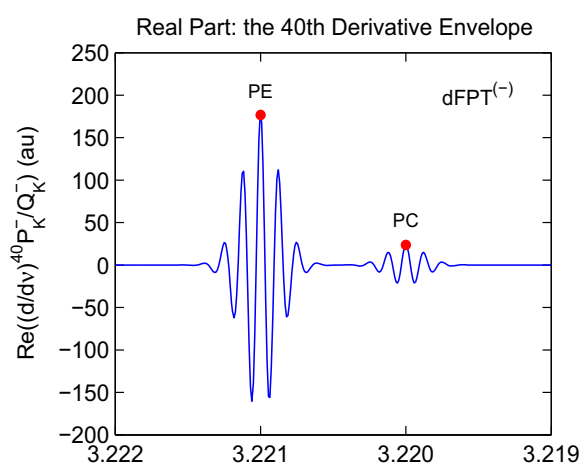

(b) Chemical Shift (ppm)

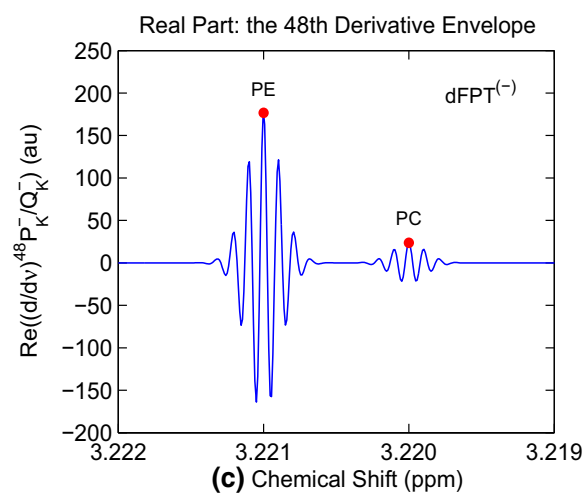

Imaginary Part: the Oth Derivative Envelope

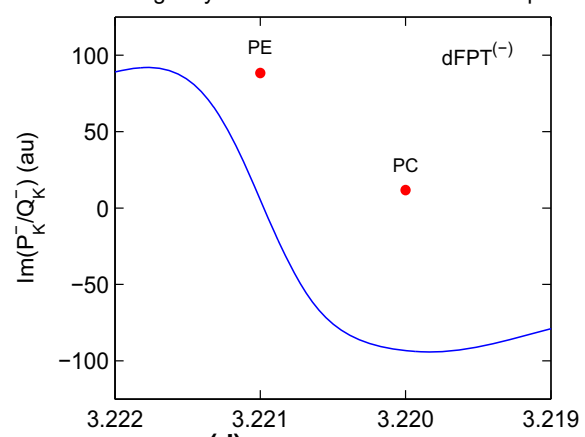

(d) Chemical Shift (ppm)

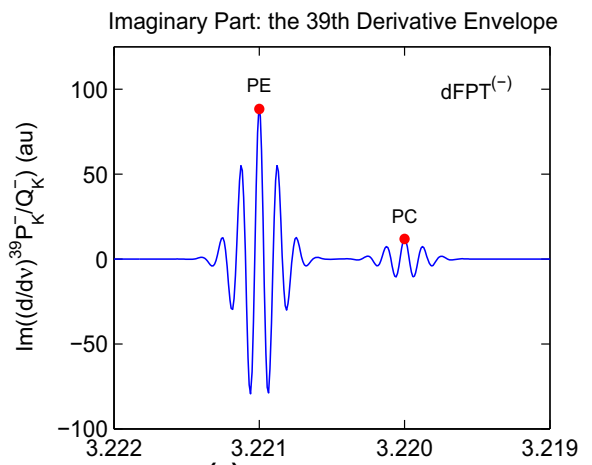

(e) Chemical Shift (ppm)

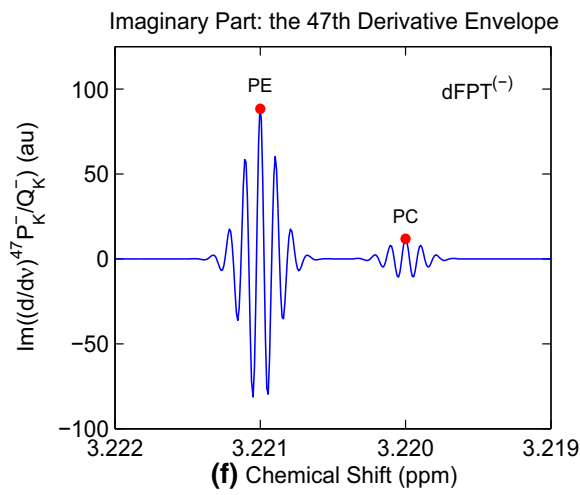

Fig. 6 Derivative fast Padé transform, $\mathrm{dFPT}^{(-)}$, for a synthesized time signal sampled by using $c_{n}$ from (4) and the spectral parameters (80) according to the FIDs encoded by in vitro MRS from excised human breast cancer tissue, as per Ref. [29]. The left and right columns are respectively for the real $(m=0,40,48)$ and imaginary $(m=0,39,47)$ parts of complex non-derivative and derivative envelopes. Filled circles are the input peak heights $h_{k}$. (Color online) 
Nonparametric Quantification: dMRS; Synthesized Noiseless FIDs, Input Peak Heights (Filled Circles)

Real Parts and Magnitudes of Pade Envelope \& Its Derivatives Using $\left.(\mathrm{d} / \mathrm{d} v)^{\mathrm{m}} \mathrm{P}_{\mathrm{K}}^{-} / \mathrm{Q}_{\mathrm{K}}^{-}\right) ; \mathrm{m}=0,8,16$

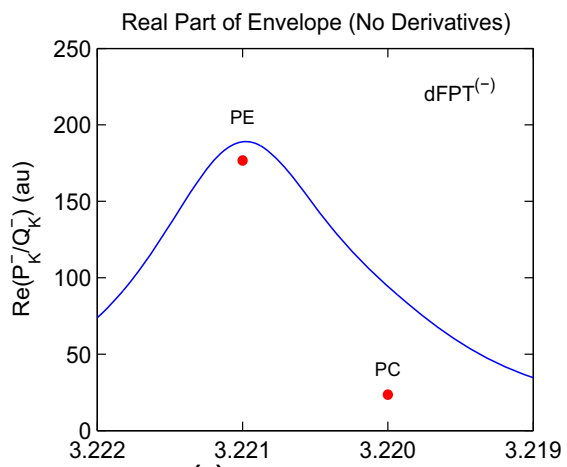

(a) Chemical Shift (ppm)

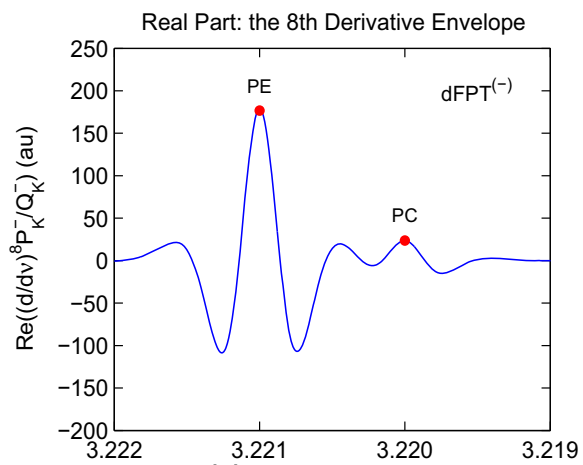

(b) Chemical Shift (ppm)

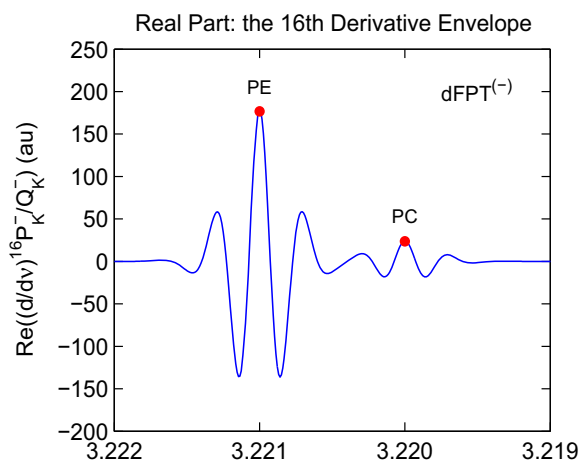

(c) Chemical Shift (ppm)

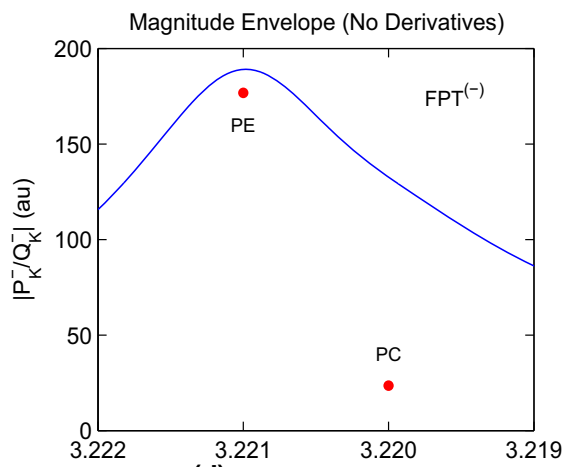

(d) Chemical Shift (ppm)

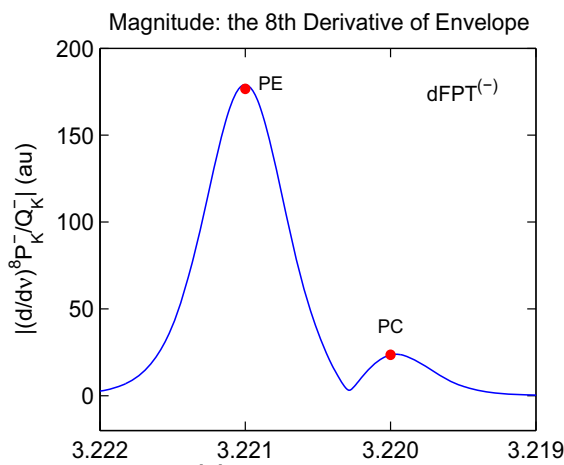

(e) Chemical Shift (ppm)

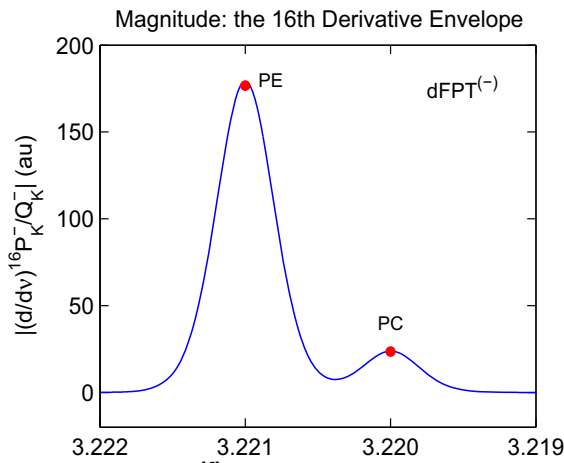

(f) Chemical Shift (ppm)

Fig. 7 Derivative fast Padé transform, $\mathrm{dFPT}^{(-)}$, for a synthesized time signal sampled by using $c_{n}$ from (4) and the spectral parameters (80) according to the FIDs encoded by in vitro MRS from excised human breast cancer tissue, as per Ref. [29]. The left and right columns are respectively for the real parts and magnitudes of complex non-derivative and derivative envelopes $(m=0,8,16)$. Filled circles are the input peak heights $h_{k}$. (Color online) 
Nonparametric Quantification: dMRS; Synthesized Noiseless FIDs, Input Peak Heights (Filled Circles)

Real Parts and Magnitudes of Pade Envelope \& Its Derivatives Using $\left.(d / d v)^{m} P_{K}^{-} / Q_{K}^{-}\right) ; m=0,24,32$

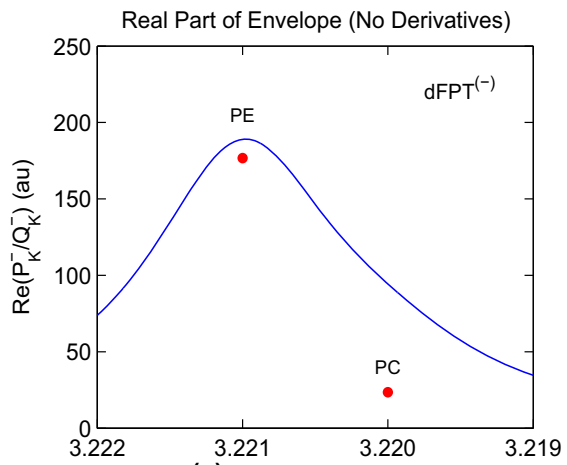

(a) Chemical Shift (ppm)

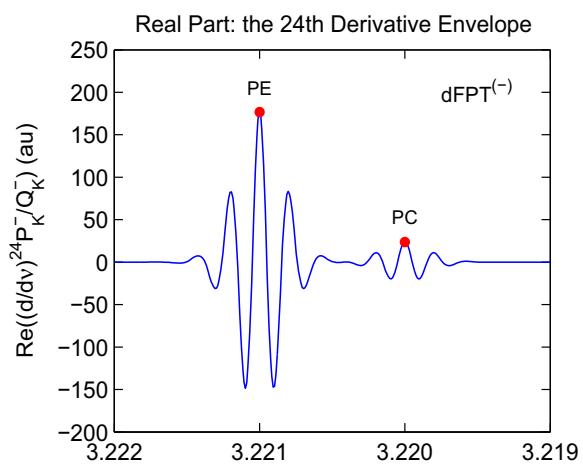

(b) Chemical Shift (ppm)

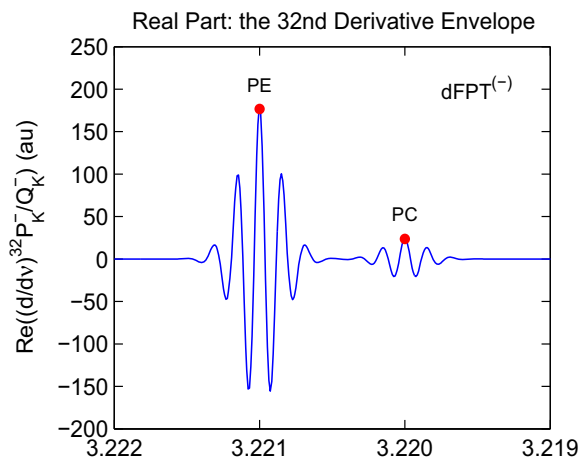

(c) Chemical Shift (ppm)

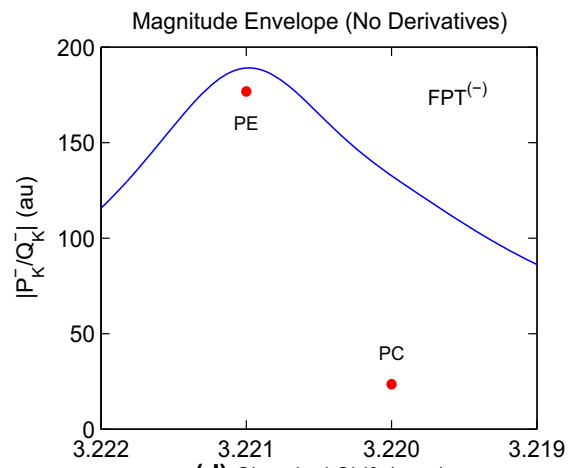

(d) Chemical Shift (ppm)

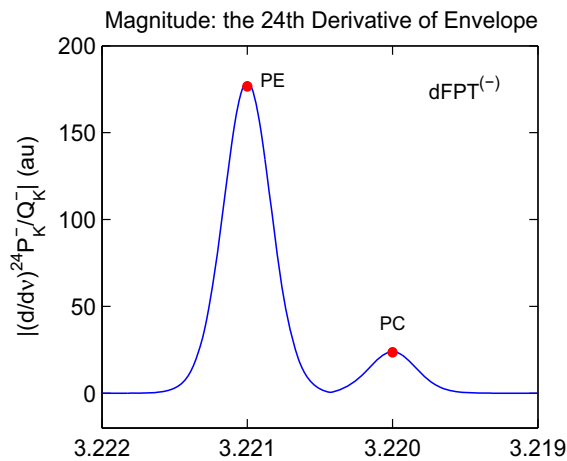

(e) Chemical Shift (ppm)

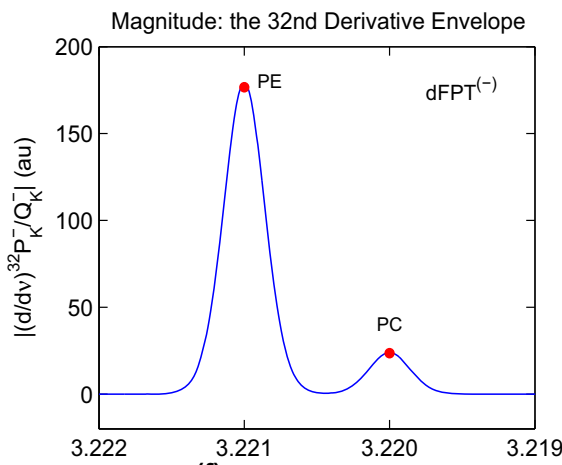

(f) Chemical Shift (ppm)

Fig. 8 Derivative fast Padé transform, $\mathrm{dFPT}^{(-)}$, for a synthesized time signal sampled by using $c_{n}$ from (4) and the spectral parameters (80) according to the FIDs encoded by in vitro MRS from excised human breast cancer tissue, as per Ref. [29]. The left and right columns are respectively for the real parts and magnitudes of complex non-derivative and derivative envelopes $(m=0,24,32)$. Filled circles are the input peak heights $h_{k}$. (Color online) 
Nonparametric Quantification: dMRS; Synthesized Noiseless FIDs, Input Peak Heights (Filled Circles)

Real Parts and Magnitudes of Pade Envelope \& Its Derivatives Using $\left.(d / d v)^{m} P_{K}^{-} / Q_{K}^{-}\right) ; m=0,40,48$

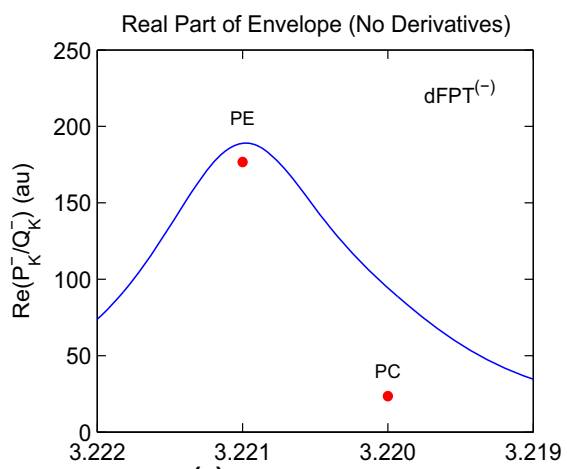

(a) Chemical Shift (ppm)

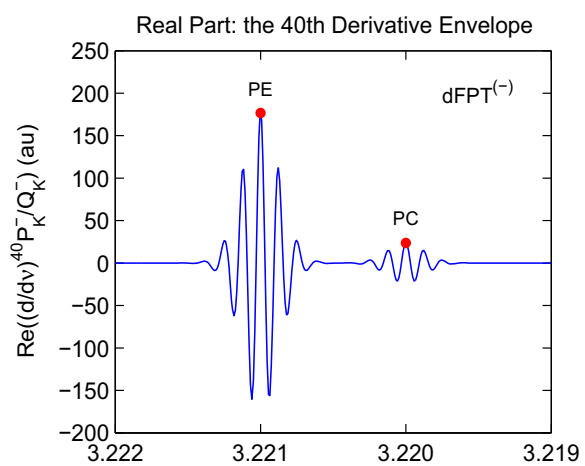

(b) Chemical Shift (ppm)

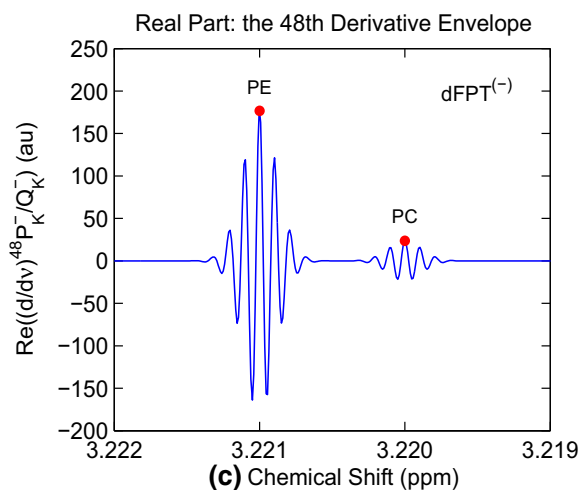

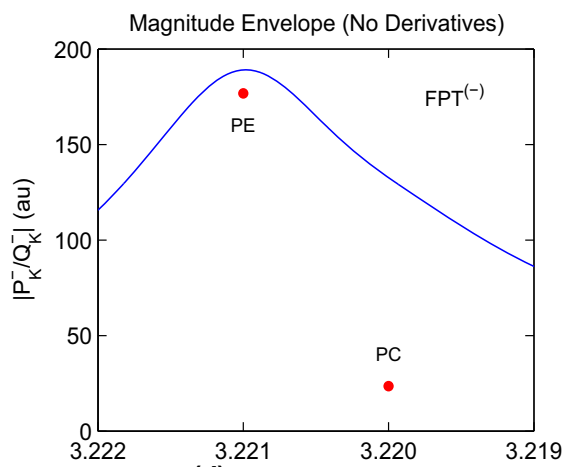

(d) Chemical Shift (ppm)

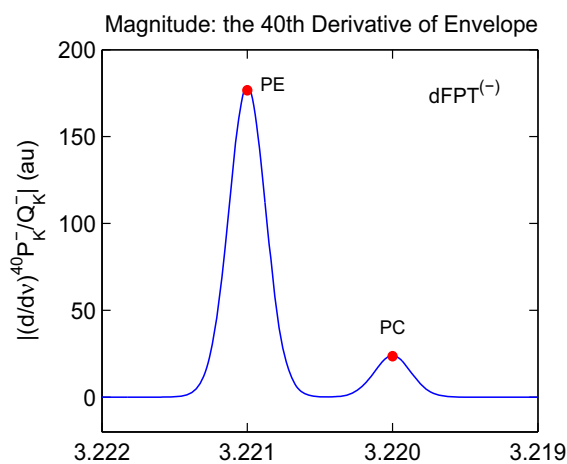

(e) Chemical Shift (ppm)

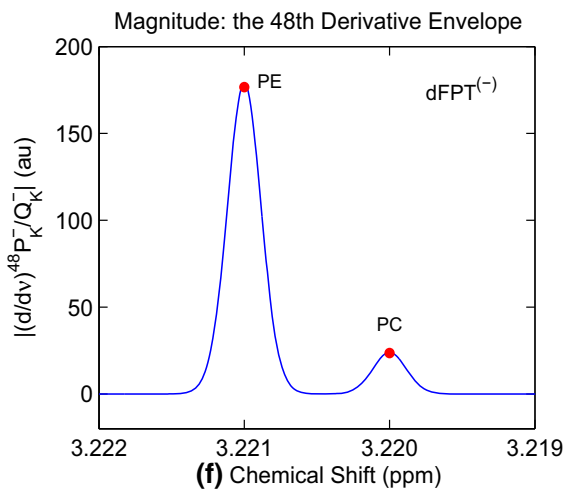

Fig. 9 Derivative fast Padé transform, $\mathrm{dFPT}^{(-)}$, for a synthesized time signal sampled by using $c_{n}$ from (4) and the spectral parameters (80) according to the FIDs encoded by in vitro MRS from excised human breast cancer tissue, as per Ref. [29]. The left and right columns are respectively for the real parts and magnitudes of complex non-derivative and derivative envelopes $(m=0,40,48)$. Filled circles are the input peak heights $h_{k}$. (Color online) 
envelopes are juxtaposed (Figs. 7-9). These latter illustrations connect the present work with Ref. [28]. In Figs. 2-9, the reference non-derivative ( $m=0)$ envelopes are also plotted. Given, as well, are the input data for the peak heights, as these represent the immediate check of the veracity of the corresponding Padé-reconstructed data.

Figures 2 and 3 show the performance of the derivative spectra $(m>0)$ relative to their non-derivative $(m=0)$ counterpart. Therein, $m$ is varied from 4 to 40 with the equidistant step of 4 , i.e. $m=0, \Delta m, 4+\Delta m, \ldots, 40$ where $\Delta m=4$. Thus, Fig. 2 deals with $m=0$ (4) 20, whereas Fig. 3 is concerned with $m=0$ and $m=$ 24 (4) 40. Here, and throughout, the variation of the derivative order $m$ is symbolized as $m=m_{\min }(\Delta m) m_{\max }$ which amounts to writing $m \in\left[m_{\min }, m_{\max }\right]$, where $\Delta m$ is the increment for augmenting the value of $m$ from its minimum $m_{\min }$ to maximum $m_{\max }$.

According to Section 3, the derivative spectral envelopes in any of the discussed modes (complex, real, imaginary, magnitude) significantly change their extremal values with the increase of the differentiation order $m$. Therefore, to directly compare, e.g. the predicted extremal value from the derivative spectra (especially in the case of higher $m$ ), normalization is required. This is achieved by normalizing the extremal values for each $m>0$ to those for $m=0$ in the entire SRI under consideration, $v \in[1.3-2.9] \mathrm{ppm}$.

The present illustration is focused on the narrow frequency range $v \in[3.205,3.290]$ ppm in which, e.g. $\max \left\{\operatorname{Re}\left(P_{K}^{-} / Q_{K}^{-}\right)\right\}$is at the location $1.332 \mathrm{ppm}$, which corresponds to lactate, Lac, as the tallest peak in the non-derivative $(m=0)$ absorption and magnitude. As such, the normalizations for Figs. 2-9 are:

$$
\begin{array}{ll}
\max \left\{\operatorname{Re}(\mathrm{d} / \mathrm{d} \nu)^{m} P_{K}^{-} / Q_{K}^{-}\right\}=\max \left\{\operatorname{Re} P_{K}^{-} / Q_{K}^{-}\right\}, & m>0, \\
\max \left\{\operatorname{Im}(\mathrm{d} / \mathrm{d} \nu)^{m} P_{K}^{-} / Q_{K}^{-}\right\}=\max \left\{\operatorname{Im} P_{K}^{-} / Q_{K}^{-}\right\}, & m>0, \\
\max \left\{\left|(\mathrm{d} / \mathrm{d} \nu)^{m} P_{K}^{-} / Q_{K}^{-}\right|\right\}=\max \left\{\left|P_{K}^{-} / Q_{K}^{-}\right|\right\}, & m>0 .
\end{array}
$$

We emphasize that since this normalization is done only for the tallest peak in the spectrum, the correct reconstructions would be only those which predict all the input data for all the other peak heights, as well as the peak positions and peak breadths.

It is seen that the normalized maximal values of the derivative envelopes in their real (Figs. 2-9), imaginary (Figs. 4-6) and magnitude modes (Figs. 7-9) exactly predict the input peak heights, marked as small filled circles. Recall that the nonderivative $(m=0)$ dispersion (the imaginary part of the complex spectrum) form panel (a) of Fig. 1 was amenable to extraction of the same fundamental parameters typically attributed to the corresponding absorption as the real part of the same complex spectrum for $m=0$. This feature should by no means be restricted to the case with $m=0$ alone. The right columns in Figs. 4-6 for the imaginary parts of the highorder derivative envelopes also predict all the correct peak heights $h_{k}$ of the nonderivative $(m=0)$ absorption. The remaining input parameters $\left(\operatorname{Re} v_{k}, \operatorname{Im} v_{k},\left|d_{k}\right|\right)$ are extractable as well from these imaginary derivative spectra by reference to the pertinent connecting expressions derived similarly to Sections 2 and 3.

Figures 7-9 compare the derivative spectra in the modes of the real parts and magnitudes of the corresponding derivative complex envelopes. It is noted here that 
the predicted normalized peak heights of both mentioned modalities are the same and that they all coincide with the associated input data for $h_{k}$. The other input fundamental parameters are retrieved from the lineshapes in Figs. 7-9 along the procedure similar to that explained for Figs. 2-6.

As seen in the illustrations, the real and imaginary derivative spectra exhibit a number of spectral structures. For example, the real part of the $m$ th derivative spectrum possesses $m$ zeros and $m+1$ maximae (positive peaks) or minimae (negative peaks, i.e. dips). Moreover, for the real and imaginary parts of the $m$ th derivative spectrum with $m$ even and odd, respectively, the strongest maximum is always located at the resonance frequency $\zeta_{k}$ and the sidelobes are placed symmetrically around $\zeta_{k}$. Specifically, in Figs. 2-9, only a handful of these sidelobes appear, whereas most of them are invisible due to their small intensities. On the other hand, for any given $m$, the magnitude spectrum has no sidelobes, since their underlying real and imaginary parts cancel out. In other words, the magnitude mode of the $m$ th derivative spectrum, being strictly positive-definite, has a single peak per component at each resonance chemical shift. From the supplied analytical expressions, the fundamental peak parameters of the non-derivative $(m=0)$ absorption mode can be deduced directly from those for the magnitudes of the $m$ th derivative spectra with $m>0$.

\section{Discussion}

\subsection{General aspects of derivative spectroscopy}

The derivative fast Padé transform, dFPT, has recently been introduced [28] as a super-high-resolution signal processor for exact quantification with reliance upon reconstruction of total shape spectra (envelopes) alone. No fitting of envelopes is employed. Instead, the non-parametrically computed envelopes in the customary nonderivative fast Padé transform, FPT, are subjected to the differentiation operators to yield the dFPT. The non-parametric envelope in the FPT is the quotient of two frequency-dependent polynomials $P_{L} / Q_{K}$ whose expansion coefficients are uniquely determined only in terms of the input original (i.e. unedited) time signal points. Application of the differentiation operator $(\mathrm{d} / \mathrm{d} \nu)^{m}$ to the envelope $P_{L} / Q_{K}$ yields the derivative envelope $(\mathrm{d} / \mathrm{d} \nu)^{m} P_{L} / Q_{K}$ in the dFPT. The derivative order $m$ is a nonnegative integer and $v$ is the sweep linear frequency. Mathematically, $(\mathrm{d} / \mathrm{d} v)^{m} P_{L} / Q_{K}$ is an analytical expression for any chosen $m$ because $P_{L} / Q_{K}$ is itself also a closed formula. Specifically, the derivative envelope $(\mathrm{d} / \mathrm{d} v)^{m} P_{L} / Q_{K}$ in the FPT is the Bell polynomial. This latter derivative response function, due to the seed spectrum $P_{L} / Q_{K}$, is a linear combination of rational polynomials that, hence, also belong to the category of the Padé approximants. Importantly, in this latter superposition, each rational polynomial has $Q_{K}^{l}(l=1,2, \ldots, m)$ in their denominators. This implies that the derivative envelope $(\mathrm{d} / \mathrm{d} v)^{m} P_{L} / Q_{K}$ in the dFPT will possess the same polar structure as that in the original seed spectrum $P_{L} / Q_{K}$ from the FPT. Such a circumstance permits the establishment of the relationships among the resonance quantifiers extractable from the non-parametric derivative $(m>0, \mathrm{dFPT})$ and the parametric non-derivative ( $m=0$, FPT) envelopes. The gist of the matter is that the higher-order derivative 
envelopes generated non-parametrically by lineshape processing in the dFPT can unequivocally reconstruct all the components of the input time signal, including those leading to the most tightly overlapped resonances in the reconstructed spectrum. This occurrence makes the fundamental parameters (complex resonance frequencies and amplitudes) of the time signal indeed extractable from the derivative envelopes in the dFPT. Such spectral parameters from the dFPT are found to coincide with the corresponding values obtained by explicitly solving the quantification problem in the parametric non-derivative FPT $(m=0)$. This is of utmost importance, in view of the fact that especially the tightly overlapped resonances hidden in paired or aggregated spectral structures cannot even be visualized as separate peaks, let alone quantified by any non-parametric, non-derivative signal processor, including the FPT.

The mechanism by which the non-parametric derivative envelope can uncover its masked component spectra is in the suppression or elimination of the interference effects among the constituent resonances. The derivative transform $(\mathrm{d} / \mathrm{d} v)^{m}$ in the dFPT makes the component peaks thinner and taller, relative to the non-derivative seed envelope $P_{L} / Q_{K}$. This is simultaneously coupled with the flattening of the peak baselines in the derivative envelopes $(\mathrm{d} / \mathrm{d} v)^{m} P_{L} / Q_{K}$. Thinner peaks amount to the resolution enhancement of the derivative versus non-derivative envelopes. Flattened peak baselines imply noise suppression. Therefore, signal-to-noise ratios (SNR) are improved when passing from the non-derivative to derivative envelopes.

All the expounded facets of the dFPT are jointly confirmed in Ref. [28] and in the present study. In the current work, the explicit analytical expressions are given, connecting the resonance parameters from the non-parametric dFPT and the exact components of the input time signal. These formulae relate the component lineshapes of derivative spectra to the non-derivative absorption mode of the individual resonances. This is prioritized because the absorptive component spectra have the most straightforward interpretation, in terms of the peak positions, peak widths and peak heights. These latter parameters also provide the peak areas. The special significant of the peak area (particularly for magnetic resonance spectroscopy), MRS, is in its direct proportionality to the concentrations of metabolites detected in the scanned tissue. The derivative aspects of signal processing lead to the establishment of the derivative magnetic resonance spectroscopy, dMRS. When dMRS is accompanied by the dFPT, it is anticipated that the ensuing enhanced resolution and increased SNR will be of critical importance for dMRS. It is precisely the resolution and SNR improvements that are most needed in the use of magnetic resonance spectroscopy within clinical diagnostics.

\subsection{Derivative magnetic spectroscopy as a practical strategy: Its potential to aid in cancer diagnostics}

Magnetic resonance spectroscopy, MRS, and spectroscopic imaging, MRSI, have provided noteworthy improvements in cancer diagnostics compared to anatomical imaging via MRI, as reviewed e.g. in Refs. [2,26,33-36]. Notwithstanding these important achievements, the diagnostic accuracy of MRS and MRSI is still generally insufficient for the stringent requirements of personalized cancer medicine 
$[10,12,37,38]$. In some applications, notably for timely and accurate detection of ovarian cancer, MRS and MRSI have heretofore failed to provide sufficient added value to be applied in clinical practice [16,39]. The main reason for this inadequacy has been reliance upon ambiguous methods for analyzing encoded MRS data, based upon Fourier processing and eventual subsequent fitting. This deficiency has been overcome by the parametric, quantification-equipped fast Padé transform, FPT, which has been extensively validated for processing MRS time signals encoded in vivo or associated with cancerous tissues, as well as with benign pathology that can be difficult to distinguish from malignancy $[2,8-12,14-16,40]$. As a complement to the parametric FPT, the non-parametric dFPT provides a strategy that would be of immediate practical importance in the clinical setting. Namely, the complete clinical MRS information would be directly visualized and quantified using the dFPT. In particular, overlapping peaks would be fully resolved by the dFPT and the key peak parameters: position, full-width at half maximum, and peak height could be directly read off from the plotted lineshapes. The concentrations of all the diagnostically relevant metabolites would then become immediately accessible to the clinician.

This derivative spectroscopy would greatly facilitate implementation of MRSI, which is most often needed within cancer diagnostics, to provide volumetric coverage. Through MRSI the chemical specificity of MRS is merged with the spatial localization techniques of MRI to provide multiple MRS signals over a volume of interest of tissue [41,42]. The major challenge entailed in MRSI is that thousands of noisy spectra must be processed. It is fully expected that derivatives of total shape spectra via the dFPT would be most helpful for putting molecular imaging through MRSI into clinical practice.

It should also be noted that attempts have been made to use higher field scanners with the aim of improving SNR in MRS and MRSI for cancer diagnostics. These attempts were motivated by the insufficient accuracy of Fourier-based in vivo MRS and MRSI with clinical (1.5 or 3T) MR scanners for identifying cancers and distinguishing these from benign lesions [43-45]. However, at higher field strengths, measured linewidths are generally wider due to increased susceptibility effects [46]. Overall, the use of higher field scanners has frequently failed to provide the anticipated improved cancer detection [26,43-45]. Moreover, the enormous costs [47] would preclude such an approach for widespread applications, especially in screening/surveillance. In sharp contrast, via dFPT-based dMRS and dMRSI, improved SNR is achieved together with narrowing of the linewidths. This efficient approach is anticipated to be applicable on standard clinical scanners $(1.5 \mathrm{~T})$, and would thus offer the further benefit of costeffectiveness.

In the present study and in Ref. [28] on dMRS, the dFPT was applied to time signals akin to those encoded from breast cancer, according to Ref. [29]. The clinical-public health importance was a major motivation for selecting this problem, especially in light of the positive impact of timely breast cancer detection upon survival [48-50]. The breast cancer biomarker phosphocholine, PC, which very closely overlaps with phosphoethanolamine, PE, was identified and exactly quantified by non-parametric analysis through the dFPT.

Notably, PC is also a biomarker of other cancers [30-32], possibly due to loss of tumor suppressor p53 function [51]. Dense spectra with numerous other overlap- 
ping resonances of diagnostic importance are also seen in brain tumors, prostate, lung, ovarian and other cancers $[10,14,16,19,52]$. Synergistic development has thus far been witnessed, whereby each step forward with Padé-optimized MRS in cancer diagnostics, informs and advances other areas [19]. With non-parametric quantification using derivative spectra in the dFPT, we anticipate that the implementation of in vivo encoded dMRS and dMRSI for early cancer diagnostics as well as for other aspects of personalized cancer care will soon be forthcoming.

\section{Conclusions and perspectives}

The present study expands further our earlier investigation of derivative signal processing applicable to all spectroscopic methods in vastly different fields. It is the versatile resonance effect, as a physical phenomenon, which brings many seemingly unrelated problem areas under a common umbrella. Resonance is chiefly manifested in a sudden pattern change through an abrupt switch of the spectral lineshape profile from its rising to a falling behavior. In an absorption spectrum, this appears as a peak whose top (maximum) is centered precisely at the resonance frequency. The measure of the spread of the lineshape in the absorption mode is the profile breadth called the full width at half maximum. Thus, to quantify an absorptive resonance, it suffices to reconstruct the peak position, width and height.

For a pure absorption, the resonance phases is equal to zero and, thus, a typical profile encountered across inter-disciplinary fields, is a bell-shaped symmetric Lorentzian spectral lineshape. The area underneath such a peak is proportional to the number of species responsible for generating the resonance effect. Such a proportionality is direct in magnetic resonance spectroscopy, MRS, which, due to this occurrence, informs directly about the concentration of metabolites from the scanned tissue of the patient in clinical diagnostics. This is very important compared to some other spectroscopies necessitating more quantities (e.g. molar extinction coefficients) to assess the abundance of the active species that produced the detectable resonance signal. Overall, for unambiguous interpretation of spectra in different modes, it is always necessary to relate these profiles to purely absorptive lineshapes.

In reality, however, dealing with experimental measurements such as encoded time signals, the computed spectra are mixtures of absorptive and dispersive lineshapes. This occurs because the phases of the constituent resonances are non-zero, and, moreover, they may widely differ from each other across the entire range $[-\pi,+\pi]$. Absorption and dispersion are out of phase, in the sense that their forms, just like the sine and cosine trigonometric functions, are mismatched due to an angle shift of $\pi / 2$. The absorption-dispersion interference distorts the spectral profile relative to the symmetric bell-shape Lorentzians. This obstacle constitutes the so-called "phase-shift" problem which impacts detrimentally onto the quantification as well as interpretation of spectra and/or the underlying time signals. A number of sources can yield the phase shift impediment to adequate spectral evaluation and estimation. For example, in localized MRS, vibrations of the gradient coils at acoustic frequencies can produce a time-varying magnetic field added to the static magnetic field. The resulting frequency modulation affects the resonance phases in the encoded time signal. More- 
over, the encoded signal phase is altered by a time delay between switching the radio frequency excitation pulse and the beginning of the data acquisition. In addition to these and other disturbances, the omnipresent stochastic noise in measurements can modify all the fundamental resonant phases.

The just emphasized fact that the interference effect among resonances can be mediated by non-zero phases provides a guide to tackle the enumerated problems whose solutions could, in turn, simultaneously improve resolution and signal-to-noise ratio, SNR. This indicates that interference of resonances could be reduced by weakening the role of phases. Such a weakening would diminish the chance of artefacts caused by phase shifts among resonances. Moreover, reduction of interference of resonances would improve resolution. The reason is that weaker interactions of resonances would allow their clearer separation. Most importantly, this would increase the possibility of disentangling overlapped resonances hidden in compound spectral structures. The latter possiblity is the main task of all spectroscopies, including MRS, whose encoded time signals yield spectra abundant with unresolved resonances. Due to nonuniqueness, no meaningful metabolite assignment can be attributed by non-parametric processing to any unsplit resonance buried in aggregated lineshape profiles.

In order to be implemented, the outlined descriptive guide needs an appropriate logistics, and the question arises as to which of the mathematical operations would be pertinent. The sought operation should be sensitive to detecting subtle changes, invisible in an overall envelope comprised of a number of different components. A visual manifestation of such changes in a seemingly smooth curve is the slope alteration. A slope change in a curve corresponds to the physical notion of the rate of occurrence of an elementary event in a composite phenomenon. The first-order rates are mathematically described by the first derivative operation of a given function with respect to its independent variable. In MRS, such composite functions are total shape spectra or envelopes (comprised of a number of components associated with metabolites), and the independent variable is the linear sweep frequency $v$. Derivative transform $(d / d v)^{m}$ for non-negative integers $m$ applied to a given spectrum leads to derivative spectroscopies, such as the derivative MRS, i.e. dMRS.

It is shown that dMRS becomes an especially powerful diagnostic modality if teamed with the derivative fast Padé transform, dFPT. In the dFPT, the spectral envelope is in the form $(\mathrm{d} / \mathrm{d} v)^{m} P_{L}(v) / Q_{K}(v)$. Here, the seed (non-derivative, $m=0$ ) spectrum is the quotient $P_{L}(v) / Q_{K}(v)$ of the numerator $P_{L}$ and the denominator $Q_{K}$ polynomials. It is demonstrated that all the mentioned problems are simultaneously solved by the dFPT. Such an achievement widens the range of applications of the non-parametric dFPT relative to the non-parametric FPT. In particular, the dFPT reduces resonance interference effects by weakening the phase influences. This diminishes the chance for lineshape distortions caused by phase shifts among the constituent resonances of an envelope. Moreover, with increasing derivative order $m$, the peaks become thinner, and their baseline systematically flattens. As a result, both resolution and signal-to-noise ratio, SNR, are improved.

In the dFPT, both the seed $P_{L} / Q_{K}$ and derivative $(\mathrm{d} / \mathrm{d} \nu)^{m} P_{L} / Q_{K}$ spectra are generated non-parametrically, i.e. without solving the quantification problem. Yet, all the fundamental parameters (frequencies and amplitudes) are reconstructed by the dFPT. This is done by using the presently listed expressions that connect the resonance 
parameters of any $m$ th derivative lineshape $(m>0)$ and the non-derivative $(m=0)$ absorption profile.

Overall, by the exclusive reliance upon lineshape estimation, the dFPT reconstructs the correct peak positions, widths and heights of all the physical resonances, including those that are tightly overlapped and remain unresolved in all the non-parametric or shape estimators, including the FPT. It is anticipated that the dFPT will find useful applications in all spectroscopies. This is presently evidenced for the case of derivative magnetic resonance spectroscopy, dMRS, whose overall performance is expected to be significantly improved when signal processing is carried out by the derivative fast Padé transform, dFPT.

Acknowledgements This work was supported by King Gustav the 5th Jubilee Fund, The Marsha Rivkin Center for Ovarian Cancer Research and FoUU through Stockholm County Council to which the authors are grateful.

Open Access This article is distributed under the terms of the Creative Commons Attribution 4.0 International License (http://creativecommons.org/licenses/by/4.0/), which permits unrestricted use, distribution, and reproduction in any medium, provided you give appropriate credit to the original author(s) and the source, provide a link to the Creative Commons license, and indicate if changes were made.

\section{References}

1. Dž. Belkić, Quantum-Mechanical Signal Processing and Spectral Analysis (Institute of Physics Publishing, Bristol, 2005)

2. Dž. Belkić, K. Belkić, Signal Processing in Magnetic Resonance Spectroscopy with Biomedical Applications (Taylor \& Francis Publishers, London, 2010)

3. J.W. Cooley, J.W. Tukey, An algorithm for the machine calculation of complex Fourier series. Math. Comput. 19, 297-301 (1965)

4. J.W.C. van der Veen, R. de Beer, P.R. Luyten, D. van Ormondt, Accurate quantification of in vivo 31P NMR signals using the variable projection method and prior knowledge. Magn. Reson. Med. 6, 92-98 (1988)

5. L. Vanhamme, A. van den Boogaart, S. van Haffel, Improved method for accurate and efficient quantification of MRS data with use of prior knowledge. J. Magn. Reson. 129, 35-43 (1997)

6. S.W. Provencher, Estimation of metabolite concentrations from localized in vivo proton NMR spectra. Magn. Reson. Med. 30, 672-679 (1993)

7. W.W.F. Pijnappel, A. van den Boogaart, R. de Beer, D. van Ormondt, SVD-based quantification of magnetic resonance signals. J. Magn. Reson. 97, 122-134 (1992)

8. Dž. Belkić, K. Belkić, Mathematical modeling applied to an NMR problem in ovarian cancer detection. J. Math. Chem. 43, 395-425 (2008)

9. Dž. Belkić, K. Belkić, Resolution enhancement as a key step towards clinical implementation of Padéoptimized magnetic resonance spectroscopy for diagnostic oncology. J. Math. Chem. 51, 2608-2637 (2013)

10. Dž. Belkić, K. Belkić, The fast Padé transform for noisy magnetic resonance spectroscopic data from the prostate: Potential contribution to individualized prostate cancer care. J. Math. Chem. 54, 707-764 (2016)

11. Dž. Belkić, K. Belkić, Proof-of-the-concept study on mathematically optimized magnetic resonance spectroscopy for breast cancer diagnostics. Technol. Cancer Res. Treat. 14, 277-297 (2015)

12. Dž. Belkić, K. Belkić, Mathematically-optimized magnetic resonance spectroscopy in breast cancer diagnostics: Implications for personalized cancer medicine. J. Math. Chem. 54, 186-230 (2016)

13. Dž. Belkić, K. Belkić, Quantification by the fast Padé transform of magnetic resonance spectroscopic data encoded at $1.5 \mathrm{~T}$ : implications for brain tumor diagnostics. J. Math. Chem. 54, 602-655 (2016)

14. Dž. Belkić, K. Belkić, Improving the diagnostic yield of magnetic resonance spectroscopy for pediatric brain tumors through mathematical optimization. J. Math. Chem. 54, 1461-1513 (2016) 
15. Dž. Belkić, K. Belkić, Iterative averaging of spectra as a powerful way of suppressing spurious resonances in signal processing. J. Math. Chem. 55, 304-348 (2017)

16. Dž. Belkić, K. Belkić, In vivo magnetic resonance spectroscopy for ovarian cancer diagnostics: Quantification by the fast Padé transform. J. Math. Chem. 55, 349-405 (2017)

17. Dž Belkić, K. Belkić, Synergism of spectra averaging and extrapolation for quantification of in vivo MRS time signals encoded from the ovary. J. Math. Chem. 55, 1067-1109 (2017)

18. Dž. Belkić, K. Belkić, Encoded in vivo time signals from the ovary in magnetic resonance spectroscopy: poles and zeros as the cornerstone for stability of response functions of systems to external perturbations. J. Math. Chem. 55, 1110-1157 (2017)

19. Dž. Belkić, K. Belkić, Robust high-resolution quantification of time signals encoded by in vivo magnetic resonance spectroscopy. Nucl. Instr. Meth. Phys. Res. A 878, 99-128 (2018)

20. A.C. Ojo, The Analysis and Automatic Classification of Nuclear Magnetic Resonance Signals. Ph.D. Thesis, The University of Edinburgh (2010). Edinburgh Research Archive (http://hdl.handle.net/1842/ 4109)

21. J.M. Zhang, Human brain glutamate, glutamine, $\gamma$-aminobutyric acid: Proton magnetic resonance spectral quantification with the fast Padé transform. PhD Thesis. University of California Los Angeles (2013). (scholarship.org/uc/item/1np12339)

22. D.C. Williamson, H. Hawesa, N. Thacker, S.R. Williams, Robust quantification of short echo time $1 \mathrm{H}$ magnetic resonance spectra using the Padé approximant. Magn. Reson. Med. 55, 762-771 (2006)

23. S. Williams, Dž. Belkić, K. Belkić, Potential and obstacles of MRS in the clinical setting, in Magnetic Resonance Imaging and Spectroscopy, Volume 3, Comprehensive Biomedical Physics, ed. by Dž. Belkić, K. Belkić (Elsevier, Amsterdam, 2014), pp. 315-329

24. K. Belkić, Inter-disciplinarity of MR and future perspectives with a focus on screening, in Magnetic Resonance Imaging and Spectroscopy, Volume 3, Comprehensive Biomedical Physics, ed. by Dž. Belkić, K. Belkić (Elsevier, Amsterdam, 2014), pp. 417-433

25. Dž. Belkić, Mathematically-optimized MR reconstructions, in Magnetic Resonance Imaging and Spectroscopy, Volume 3, Comprehensive Biomedical Physics, ed. by Dž. Belkić, K. Belkić (Elsevier, Amsterdam, 2014), pp. 399-416

26. Dž. Belkić, K. Belkić, Visualizing hidden components of envelopes non-parametrically in magnetic resonance spectroscopy: Phosphocholine, a breast cancer biomarker. J. Math. Chem. 55, 1698-1723 (2017)

27. Dž. Belkić, K. Belkić, Robust identification of the cancer biomarker phosphocholine through partitioned envelopes in noisy magnetic resonance spectroscopic data by the non-parametric fast Padé transform. J. Math. Chem. 55, 2004-2047 (2017)

28. Dž. Belkić, K. Belkić, Exact quantification by the nonparametric fast Padé transform using only shape estimation of high-order derivatives of envelopes. J. Math. Chem. 56, 268-314 (2018)

29. I.S. Gribbestad, B. Sitter, S. Lundgren, J. Krane, D. Axelson, Metabolite composition in breast tumors examined by proton nuclear magnetic resonance spectroscopy. Anticancer Res. 19, 1737-1746 (1999)

30. K. Glunde, J. Jiang, S.A. Moestue, I.S. Gribbestad, MRS/MRSI guidance in molecular medicine: targeting choline and glucose metabolism. NMR Biomed. 24, 673-690 (2011)

31. E. Iorio, D. Mezzanzanica, P. Alberti, F. Spadaro, C. Ramoni, S. D’Ascenzo, D. Millimaggi, A. Pavan, V. Dolo, S. Canavari, F. Podo, Alterations of choline phospholipid metabolism in ovarian tumor progression. Cancer Res. 65, 9369-9376 (2005)

32. N.P. Davies, M. Wilson, L.M. Harris, K. Natarajan, S. Lateef, L. MacPherson, S. Sgouros, R.G. Grundy, T. Arvanitis, A. Peet, Identification and characterization of childhood cerebellar tumors by in vivo proton MRS. NMR Biomed. 21, 908-918 (2008)

33. M. Castillo, History and evolution of brain tumor imaging: insights through radiology. Radiology 273(Suppl), S111-S125 (2014)

34. W. Hollingworth, L. Medina, R. Lenkinski, D. Shibata, B. Bernal, D. Zurakowski, B. Comstock, J. Jarvik, Systematic review of MRS for the characterization of brain tumors. Am. J. Neuroradiol. 27, 1404-1411 (2006)

35. V. Kumar, N. Jagannathan, S. Thulkar, R. Kumar, Prebiopsy MRS and imaging in the diagnosis of prostate cancer. Int. J. Urol. 19, 602-613 (2012)

36. J.K. Begley, T.W. Redpath, F.J. Gilbert, In vivo proton MRS of breast cancer: a review of the literature. Breast Cancer Res. 14, 207 (10 pages) (2012)

37. Dž. Belkić, K. Belkić, Molecular imaging in the framework of personalized cancer medicine. Isr. Med. Assoc. J. 15, 665-672 (2013) 
38. Dž. Belkić, K. Belkić, The role of optimized molecular imaging in personalized cancer medicine. Diag. Imaging Eur. 30, 28-31 (2014)

39. K. Belkić, Dž. Belkić, The challenge of ovarian cancer: steps towards early detection through advanced signal processing in MRS. Isr. Med. Assoc. J. 19, 517-525 (2017)

40. Dž. Belkić, K. Belkić, Padé-optimization of noise-corrupted magnetic resonance spectroscopic time signals from fibroadenoma of the breast. J. Math. Chem. 52, 2680-2713 (2014)

41. R.W. Prost, Magnetic resonance spectroscopic imaging, in Magnetic Resonance Imaging and Spectroscopy, Volume 3, Comprehensive Biomedical Physics, ed. by Dž. Belkić, K. Belkić (Elsevier, Amsterdam, 2014), pp. 331-345

42. S.J. Nelson, E. Graves, A. Pirzkall, X. Li, A. Antiniw Chan, D.B. Vigneron, T.R. McKnight, In vivo molecular imaging for planning radiation therapy of gliomas: an application of $1 \mathrm{H}$ MRSI. J. Magn. Reson. Imaging 16, 464-476 (2002)

43. V.O. Boer, B.L. Bank, G. van Vliet, P. Luijten, D. Klomp, Direct $B 0$ field monitoring and read-time $B 0$ field updating in the human breast at 7 Tesla. Magn. Reson. Med. 67, 586-591 (2012)

44. I. Dimitrov, D. Douglas, J. Ren, N. Smith, A. Webb, A. Sherry, C. Malloy, In vivo determination of human breast fat composition by $1 \mathrm{H}$ magnetic resonance spectroscopy at 7T. Magn. Reson. Med. 67, 20-26 (2012)

45. P.J. Bolan, S. Meisamy, E. Baker, J. Lin, T. Emory, M. Nelson, L. Everson, D. Yee, M. Garwood, In vivo quantification of choline compounds in the breast with 1H MR spectroscopy. Magn. Reson. Med. 50, 1134-1143 (2003)

46. M. McLean, Fundamentals of MR spectroscopy, in Magnetic Resonance Imaging and Spectroscopy, Volume 3 Comprehensive Biomedical Physics, ed. by Dž. Belkić, K. Belkić (Elsevier, Amsterdam, 2014), pp. 257-271

47. M.E. Ladd, High versus low static magnetic fields in MRI, in Magnetic Resonance Imaging and Spectroscopy, Volume 3 Comprehensive Biomedical Physics, ed. by Dž. Belkić, K. Belkić (Elsevier, Amsterdam, 2014), pp. 55-68

48. R.A. Smith, K.S. Andrews, D. Brooks, S.A. Fedewa, D. Manassaram-Baptiste, D. Saslow, O.W. Brawley, R.C. Wender, Cancer screening in the United States, 2017: A review of current American Cancer Society Guidelines and current issues in cancer screening. CA: Cancer J. Clin. 67, 100-121 (2017)

49. L. Pace, N. Keating, A systematic assessment of benefits and risks to guide breast cancer screening decisions. J. Am. Med. Assoc. 311, 1327-1335 (2014)

50. S. Njor, W. Schwartz, M. Blicert-Toft, E. Lynge, Decline in breast cancer mortality: How much is attributable to screening? J. Med. Screen. 22, 20-27 (2015)

51. N. Mori, R. Delsite, K. Natarajan, M. Kulawiec, Z. Bhujwalla, K. Singh, Loss of p53 function in colon cancer cells results in increased phosphocholine and total choline. Molec. Imaging 3, 319-323 (2004)

52. W. Chen, S. Lu, J. Ou, G. Wang, Y. Zu, F. Chen, C. Bai, Metabonomic characteristics and biomarker research of human lung cancer tissues by HR 1H NMR spectroscopy. Cancer Biomark. 16, 653-664 (2016) 